\title{
A set of top quark spin correlation and polarization observables for the LHC: Standard Model predictions and new physics contributions
}

\author{
Werner Bernreuther, ${ }^{a, 1}$ Dennis Heisler ${ }^{a}$ and Zong-Guo Si ${ }^{b}$ \\ ${ }^{a}$ Institut für Theoretische Teilchenphysik und Kosmologie, RWTH Aachen University, \\ Aachen, 52056 Germany \\ ${ }^{b}$ School of Physics, Shandong University, Jinan, \\ Shandong, 250100 China \\ E-mail: breuther@physik.rwth-aachen.de, \\ heisler@physik.rwth-aachen.de, zgsi@sdu.edu.cn
}

ABSTRACT: We consider top-antitop quark $(t \bar{t})$ production at the Large Hadron Collider (LHC) with subsequent decay into dileptonic and lepton plus jets final states. We present a set of leptonic angular correlations and distributions with which experiments can probe all the independent coefficient functions of the top-spin dependent parts of the $t \bar{t}$ production spin density matrices. We compute these angular correlations and distributions for LHC center-of-mass energies 8, 13, and $14 \mathrm{TeV}$ within the Standard Model at next-to-leading order in the QCD coupling including the mixed QCD-weak corrections and for the transverse top-quark polarization and the $t \bar{t}$ charge asymmetry also the mixed QCD-QED corrections. In addition we analyze and compute the effects of new interactions on these observables in terms of a gauge-invariant effective Lagrangian that contains all operators relevant for hadronic $t \bar{t}$ production up to mass dimension six.

Keywords: Hadronic Colliders, NLO Computations

ARXIV EPRINT: 1508.05271

\footnotetext{
${ }^{1}$ Corresponding author.
} 


\section{Contents}

1 Introduction 1

$2 t \bar{t}$ production density matrices in an orthonormal basis 3

3 Effective NP Lagrangian for hadronic top quark pair production $\quad 6$

$\begin{array}{lll}3.1 & t \bar{t} \text { production } & 6\end{array}$

3.2 Top-quark decay $\quad 9$

$\begin{array}{lll}3.3 & \text { Experimental bounds and domain of applicability } & 9\end{array}$

4 Observables and results $\quad 10$

4.1 Total $t \bar{t}$ cross section and charge asymmetry at the LHC 11

4.2 Angular distributions and correlations for dilepton and $\ell+$ jets events 14

5 Summary and conclusions $\quad 26$

$\begin{array}{ll}\text { A NP contributions to the } t \bar{t} \text { spin density matrices } & \mathbf{2 7}\end{array}$

A.1 NP contributions to $g g \rightarrow t \bar{t} \quad 28$

$\begin{array}{lll}\text { A.2 NP contributions to } q \bar{q} \rightarrow t \bar{t} & 29\end{array}$

\section{Introduction}

All available experimental data on top quarks show that this particle behaves as a bare quark that does not hadronize. This fact implies that observables associated with or induced by top-quark spin effects can be used as reliable tools for investigating the interactions of this quark. As far as hadronic top-antitop quark $(t \bar{t})$ production is concerned, the correlation of the $t$ and $\bar{t}$ spins and polarization of the $t$ and $\bar{t}$ samples in $t \bar{t}$ events were first experimentally explored at the Tevatron [1-4]. Evidence for $t \bar{t}$ spin correlations was first reported by the $\mathrm{D} \emptyset$ experiment [3] and they were first observed by the ATLAS experiment [5]. The ATLAS and CMS experiments at the LHC (7 and $8 \mathrm{TeV})$ have performed a number of top spin correlation and polarization measurements, using various sets of observables [5-11]. These measurements have reached a level of precision that allow for a meaningful interpretation. All measurements of $t \bar{t}$ spin correlations and top-quark polarization so far are in agreement with respective Standard Model (SM) predictions [12-15].

The degree of top-quark polarization in a $t \bar{t}$ sample and the strength of the correlation of the $t$ and $\bar{t}$ spins depends on the production dynamics and, for a 'given' production dynamics, on the choice of observable, that amounts to a choice of reference axes. (These axes may be interpreted as $t$ and $\bar{t}$ spin quantization axes.) In order that future measurements at the LHC will be able to extract complete information about the $t \bar{t}$ production 
dynamics from their data as far as top-spin effects are concerned, it is desirable to devise a set of observables, in some basis, which probe all of the independent coefficient functions of the top-spin dependent parts of the $t \bar{t}$ production spin density matrices. In this paper we present such a set. The members of this set are constructed such that they have definite properties with respect to discrete symmetries. For definiteness we consider, as far as $t \bar{t}$ spin correlation effects are concerned, the dileptonic $t \bar{t}$ decay modes and, as far as polarization effects are concerned, also the lepton plus jets final states. These modes are the most sensitive channels for the investigation of top-spin effects. We compute the distributions and expectation values of these observables at next-to-leading order (NLO) QCD with respect to $t \bar{t}$ production and decay and take into account also the NLO mixed QCD weak-interaction contributions and for the transverse $t$ and $\bar{t}$ polarizations also the mixed QCD-QED corrections of order $\alpha_{s}^{2} \alpha$. In addition, we assess the sensitivity of our set of spin correlation and polarization observables to new physics (NP) effects in the framework of the effective field theory approach. We take into account all gauge invariant operators of dimension six which are relevant for hadronic $t \bar{t}$ production [16-22]. We compute the contributions of the associated anomalous couplings to our observables. There is already an extensive literature on phenomenological studies, including [23-48] and [15, 49-68, 70, 71], on how anomalous couplings, in particular, a non-zero chromo-magnetic and/or chromoelectric dipole moment of the top quark would affect $t \bar{t}$ production at hadron colliders. In fact, if one considers only the two chromo-moments and puts all other anomalous couplings to zero, it suffices to take only the experimental results for the $t \bar{t}$ cross section at the Tevatron and the LHC (7 and $8 \mathrm{TeV}$ ) and the respective NNLO QCD predictions [72] in order to obtain sensitive bounds on these two anomalous couplings [50, 55, 68]. The point we want to make with this part of our analysis is that our set of observables, including the $t \bar{t}$ charge asymmetry $^{1}$ is i) large enough to get information on all anomalous couplings of our effective Lagrangian (in fact the number of observables in this set exceeds the number of couplings) and ii) these observables allow to disentangle the various anomalous contributions to a considerable extent.

This paper is organized as follows. In the next section we decompose the $t \bar{t}$ production density matrices of the reactions $g g, q \bar{q} \rightarrow t \bar{t}$ in terms of an orthonormal basis and classify the coefficient functions of these matrices with respect to parity $\mathrm{P}, \mathrm{CP}$, naive time reversal $T_{N}$, and Bose symmetry. The decomposition applies to all parton reactions $i j \rightarrow t \bar{t} X$. In section 3 the effective Lagrangian $\mathcal{L}_{\mathrm{NP}}$ is introduced that we use for describing non-resonant new physics in hadronic $t \bar{t}$ production. We use the operator basis employed in [22], albeit with different normalization conventions for the anomalous couplings. Possible new physics contributions to the $t \rightarrow W b$ decay vertex do not affect our leptonic angular correlations and distributions defined in section 4 . We briefly recall the presently available bounds on the anomalous couplings contained in $\mathcal{L}_{\mathrm{NP}}$ and discuss the domain of applicability of the linear approximation used in section 4 . In this section, we consider $t \bar{t}$ production and decay into dileptonic and lepton plus jets final states at the LHC. For the dileptonic final states, we present a set of leptonic angular correlation variables that are constructed such that each

\footnotetext{
${ }^{1}$ The $t \bar{t}$ cross section should, of course, also be used but is, in principle, redundant; see section 4 .
} 
member of this set probes a different $t \bar{t}$ spin correlation coefficient of the production density matrices. In addition, we list a set of single lepton distributions/observables which probe the $t$ and $\bar{t}$ polarization coefficients of the production density matrices. These observables can be applied both to dileptonic and lepton plus jets final states. We compute, for $t \bar{t}$ production at the LHC at 8,13 , and $14 \mathrm{TeV}$, the two- and one-dimensional distributions

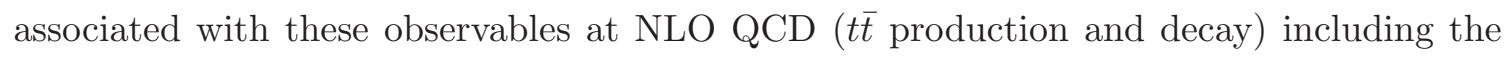
NLO mixed QCD weak-interaction corrections, for the transverse $t$ and $\bar{t}$ polarizations also the mixed QCD-QED corrections, and, in addition, the contributions induced by $\mathcal{L}_{\mathrm{NP}}$ which are linear in the anomalous couplings. For completeness we determine also the contributions of $\mathcal{L}_{\mathrm{NP}}$ to the $t \bar{t}$ cross section $\sigma_{t \bar{t}}$ and the $t \bar{t}$ charge asymmetry at the LHC, $A_{\mathrm{C}}$. For the observables considered in this paper we estimate the application range of the linear approximation in the anomalous couplings. Our results show that our set of correlation and polarisation observables, including the $t \bar{t}$ charge asymmetry $A_{\mathrm{C}}$, allows to determine all anomalous couplings of $\mathcal{L}_{\mathrm{NP}}$ and disentangles these contributions to a considerable extent. We conclude in section 5 .

\section{$2 t \bar{t}$ production density matrices in an orthonormal basis}

We consider in this section, for the purpose of setting up our notation, hadronic $t \bar{t}$ production by the $2 \rightarrow 2$ reactions

$$
\begin{aligned}
& g\left(p_{1}\right)+g\left(p_{2}\right) \rightarrow t\left(k_{1}, s_{1}\right)+\bar{t}\left(k_{2}, s_{2}\right), \\
& q\left(p_{1}\right)+\bar{q}\left(p_{2}\right) \rightarrow t\left(k_{1}, s_{1}\right)+\bar{t}\left(k_{2}, s_{2}\right),
\end{aligned}
$$

where $p_{j}, k_{j}$ and $s_{1}, s_{2}$ refer to the 4-momenta of the partons and to the spin 4-vectors of the $t$ and $\bar{t}$ quarks, respectively. The production density matrices of these reactions are defined by

$$
\begin{aligned}
R_{\alpha_{1} \alpha_{2}, \beta_{1} \beta_{2}}^{I}= & \bar{\sum}\left\langle t\left(k_{1}, \alpha_{2}\right), \bar{t}\left(k_{2}, \beta_{2}\right)|\mathcal{T}| a\left(p_{1}\right), b\left(p_{2}\right)\right\rangle^{*} \\
& \times\left\langle t\left(k_{1}, \alpha_{1}\right), \bar{t}\left(k_{2}, \beta_{1}\right)|\mathcal{T}| a\left(p_{1}\right), b\left(p_{2}\right)\right\rangle
\end{aligned}
$$

where $I \equiv a b=g g, q \bar{q}$ and the bar denotes averaging over the spins and colors of $I$ and summing over the colors of $t, \bar{t}$. Moreover, $\alpha, \beta$ are spin labels referring to $t$ and $\bar{t}$, respectively. The matrices $R^{I}$ can be decomposed in the spin spaces of $t$ and $\bar{t}$ as follows:

$$
\begin{aligned}
& R^{I}=f_{I}\left[A^{I} \mathbb{1} \otimes \mathbb{1}+\tilde{B}_{i}^{I+} \sigma^{i} \otimes \mathbb{1}+\tilde{B}_{i}^{I-} \mathbb{1} \otimes \sigma^{i}+\tilde{C}_{i j}^{I} \sigma^{i} \otimes \sigma^{j}\right], \\
& f_{g g}=\frac{\left(4 \pi \alpha_{s}\right)^{2}}{N_{c}\left(N_{c}^{2}-1\right)}, \quad f_{q \bar{q}}=\frac{\left(N_{c}^{2}-1\right)\left(4 \pi \alpha_{s}\right)^{2}}{N_{c}^{2}},
\end{aligned}
$$

where $N_{c}$ denotes the number of colors. The first (second) factor in the tensor products of the $2 \times 2$ unit matrix $\mathbb{1}$ and of the Pauli matrices $\sigma^{i}$ refers to the $t(\bar{t})$ spin space.

The functions $\tilde{B}_{i}^{I \pm}$ and $\tilde{C}_{i j}^{I}$ can be further decomposed, using an orthonormal basis which we choose as follows. Here and in the following $\hat{\mathbf{k}}$ denotes the top-quark direction of flight in the $t \bar{t}$ zero-momentum frame $(\mathrm{ZMF})$. Here $\hat{\mathbf{p}}=\hat{\mathbf{p}}_{1}$ denotes the direction of 
one of the incoming partons in this frame. Then the following set forms a right-handed orthonormal basis:

$$
\{\hat{\mathbf{r}}, \hat{\mathbf{k}}, \hat{\mathbf{n}}\}: \quad \hat{\mathbf{r}}=\frac{1}{r}(\hat{\mathbf{p}}-y \hat{\mathbf{k}}), \quad \hat{\mathbf{n}}=\frac{1}{r}(\hat{\mathbf{p}} \times \hat{\mathbf{k}}), \quad y=\hat{\mathbf{k}} \cdot \hat{\mathbf{p}}, \quad r=\sqrt{1-y^{2}} .
$$

Using rotational invariance we decompose the 3 -vectors $\tilde{\mathbf{B}}^{I \pm}$ and the $3 \times 3$ matrices $\tilde{C}_{i j}^{I}$ (which have a symmetric and antisymmetric part with 6 and 3 entries, respectively) with respect $^{2}$ to the basis (2.5):

$$
\begin{aligned}
\tilde{B}_{i}^{I \pm}= & b_{r}^{I \pm} \hat{r}_{i}+b_{k}^{I \pm} \hat{k}_{i}+b_{n}^{I \pm} \hat{n}_{i} \\
\tilde{C}_{i j}^{I}= & c_{r r}^{I} \hat{r}_{i} \hat{r}_{j}+c_{k k}^{I} \hat{k}_{i} \hat{k}_{j}+c_{n n}^{I} \hat{n}_{i} \hat{n}_{j} \\
& +c_{r k}^{I}\left(\hat{r}_{i} \hat{k}_{j}+\hat{k}_{i} \hat{r}_{j}\right)+c_{k n}^{I}\left(\hat{k}_{i} \hat{n}_{j}+\hat{n}_{i} \hat{k}_{j}\right)+c_{r n}^{I}\left(\hat{r}_{i} \hat{n}_{j}+\hat{n}_{i} \hat{r}_{j}\right) \\
& +\epsilon_{i j l}\left(c_{r}^{I} \hat{r}_{l}+c_{k}^{I} \hat{k}_{l}+c_{n}^{I} \hat{n}_{l}\right) .
\end{aligned}
$$

The coefficients $b_{v}^{I \pm}, c_{v v^{\prime}}^{I}$ are functions of the partonic c.m. energy squared, $\hat{s}$, and of $y=\hat{\mathbf{k}} \cdot \hat{\mathbf{p}}$ which is equal to the top-quark scattering angle $\cos \theta_{t}^{*}$. The terms in the antisymmetric part of (2.7) may also be represented as follows:

$$
c_{r}^{I} \epsilon_{i j l} \hat{r}_{l}=c_{r}^{I}\left(\hat{k}_{i} \hat{n}_{j}-\hat{n}_{i} \hat{k}_{j}\right), \quad c_{k}^{I} \epsilon_{i j l} \hat{k}_{l}=c_{k}^{I}\left(\hat{n}_{i} \hat{r}_{j}-\hat{r}_{i} \hat{n}_{j}\right), \quad c_{n}^{I} \epsilon_{i j l} \hat{n}_{l}=c_{n}^{I}\left(\hat{r}_{i} \hat{k}_{j}-\hat{k}_{i} \hat{r}_{j}\right) .
$$

Because the initial $g g$ state is Bose symmetric, the matrix $R^{g g}$ must satisfy

$$
R^{g g}(-\mathbf{p}, \mathbf{k})=R^{g g}(\mathbf{p}, \mathbf{k})
$$

If CP invariance holds then

$$
R_{\alpha_{1} \alpha_{2}, \beta_{1}, \beta_{2}}^{I}(\mathbf{p}, \mathbf{k})=R_{\beta_{1}, \beta_{2}, \alpha_{1} \alpha_{2}}^{I}(\mathbf{p}, \mathbf{k}), \quad I=g g, q \bar{q} .
$$

The implications of (2.9), (2.10) and of parity invariance on the structure functions defined in (2.6), (2.7) are given in table 1. In this table the properties of these functions with respect to a naive $\mathrm{T}_{\mathrm{N}}$ transformation (reversal of momenta and spins) and $\mathrm{CPT}_{\mathrm{N}}$ transformation are collected, too, where just for this purpose, the absorptive part of the respective scattering amplitude has been neglected. The entries in table 1 are to be read as follows: for example, for a $T$-invariant interaction like QCD one has $b_{n}^{I \pm}(y)=-b_{n}^{I \pm}(y)$ and thus $b_{n}^{I \pm}=0$ at Born level, whereas absorptive parts generated at 1-loop render this function non-zero. The above decomposition of the spin density matrices $R^{I}$ and classification with respect to discrete symmetries is analogous to the analysis of [73] where a non-orthogonal basis was used.

The spin density matrices of the $2 \rightarrow 3$ reactions that contribute to $t \bar{t}$ production at NLO in the gauge couplings and are inclusive in $t \bar{t}$ have the same structure as those discussed above (with coefficient functions which depend on more variables) and can also be classified with respect to discrete symmetries. Table 1 is useful as a guide for the

\footnotetext{
${ }^{2}$ The decomposition of the $\tilde{C}_{i j}^{I}$ is not unique because $\delta_{i j}=\hat{n}_{i} \hat{n}_{j}+\hat{r}_{i} \hat{r}_{j}+\hat{k}_{i} \hat{k}_{j}$.
} 


\begin{tabular}{|r|r|r|r|r|r|}
\hline & $\mathrm{CP}$ & $\mathrm{P}$ & $\begin{array}{r}\mathrm{T}_{\mathrm{N}} \\
(\mathrm{abs} \mathcal{T}=0)\end{array}$ & $\begin{array}{r}\mathrm{CPT}_{\mathrm{N}} \\
(\mathrm{abs} \mathcal{T}=0)\end{array}$ & $\begin{array}{r}\text { Bose } \\
\text { symmetry }\end{array}$ \\
\hline$A^{I}(y)$ & $A^{I}(y)$ & $A^{I}(y)$ & $A^{I}(y)$ & $A^{I}(y)$ & $A^{g g}(-y)$ \\
$b_{r}^{I \pm}(y)$ & $b_{r}^{I \mp}(y)$ & $-b_{r}^{I \pm}(y)$ & $b_{r}^{I \pm}(y)$ & $b_{r}^{I \mp}(y)$ & $-b_{r}^{g g \pm}(-y)$ \\
$b_{k}^{I \pm}(y)$ & $b_{k}^{I \mp}(y)$ & $-b_{k}^{I \pm}(y)$ & $b_{k}^{I \pm}(y)$ & $b_{k}^{I \mp}(y)$ & $b_{k}^{g g \pm}(-y)$ \\
$b_{n}^{I \pm}(y)$ & $b_{n}^{I \mp}(y)$ & $b_{n}^{I \pm}(y)$ & $-b_{n}^{I \pm}(y)$ & $-b_{n}^{I \mp}(y)$ & $-b_{n}^{g g \pm}(-y)$ \\
$c_{r r}^{I}(y)$ & $c_{r r}^{I}(y)$ & $c_{r r}^{I}(y)$ & $c_{r r}^{I}(y)$ & $c_{r r}^{I}(y)$ & $c_{r r}^{g g}(-y)$ \\
$c_{k k}^{I}(y)$ & $c_{k k}^{I}(y)$ & $c_{k k}^{I}(y)$ & $c_{k k}^{I}(y)$ & $c_{k k}^{I}(y)$ & $c_{k k}^{g g}(-y)$ \\
$c_{n n}^{I}(y)$ & $c_{n n}^{I}(y)$ & $c_{n n}^{I}(y)$ & $c_{n n}^{I}(y)$ & $c_{n n}^{I}(y)$ & $c_{n n}^{g g}(-y)$ \\
$c_{r k}^{I}(y)$ & $c_{r k}^{I}(y)$ & $c_{r k}^{I}(y)$ & $c_{r k}^{I}(y)$ & $c_{r k}^{I}(y)$ & $-c_{r k}^{g g}(-y)$ \\
$c_{r n}^{I}(y)$ & $c_{r n}^{I}(y)$ & $-c_{r n}^{I}(y)$ & $-c_{r n}^{I}(y)$ & $-c_{r n}^{I}(y)$ & $c_{r n}^{g g}(-y)$ \\
$c_{k n}^{I}(y)$ & $c_{k n}^{I}(y)$ & $-c_{k n}^{I}(y)$ & $-c_{k n}^{I}(y)$ & $-c_{k n}^{I}(y)$ & $-c_{k n}^{g g}(-y)$ \\
$c_{r}^{I}(y)$ & $-c_{r}^{I}(y)$ & $-c_{r}^{I}(y)$ & $-c_{r}^{I}(y)$ & $c_{r}^{I}(y)$ & $-c_{r}^{g g}(-y)$ \\
$c_{k}^{I}(y)$ & $-c_{k}^{I}(y)$ & $-c_{k}^{I}(y)$ & $-c_{k}^{I}(y)$ & $c_{k}^{I}(y)$ & $c_{k}^{g g}(-y)$ \\
$c_{n}^{I}(y)$ & $-c_{n}^{I}(y)$ & $c_{n}^{I}(y)$ & $c_{n}^{I}(y)$ & $-c_{n}^{I}(y)$ & $-c_{n}^{g g}(-y)$ \\
\hline
\end{tabular}

Table 1. Transformation properties of the structure functions defined in (2.6), (2.7) with respect to $\mathrm{CP}, \mathrm{P}$, and $\mathrm{T}_{\mathrm{N}}$. The implications of Bose symmetry in the last column apply only to $I=g g$.

construction of observables at the level of dileptonic and lepton plus jets final states that project onto contributions to the differential cross section which are even and odd with respect to the above discrete symmetries. These observables apply also to investigations beyond LO, cf. section 4 .

Strictly speaking, a classification of observables with respect to the discrete symmetries $\mathrm{C}$ and $\mathrm{CP}$ is not possible in proton proton collisions because the initial $p p$ state is neither an eigenstate of $\mathrm{C}$ nor of $\mathrm{CP}$. Yet, for the main $t \bar{t}$ production modes, $g g, q \bar{q} \rightarrow t \bar{t}+X$ it can be done. The 'contamination' of CP-odd observables by contributions from parity-odd mixed QCD weak contributions to $g q(\bar{q}) \rightarrow t \bar{t} q(\bar{q})$ is at the level of a few per mill [14].

We add a few remarks on the coefficient functions and on the information contained in table 1.

i) The functions $A^{I}, c_{n n}^{I}, c_{r r}^{I}, c_{k k}^{I}$, and $c_{r k}^{I}$ are generated by P- and CP-conserving interactions, in particular by QCD already at tree level. The functions $A^{I}$ determine the $t \bar{t}$ cross section, the $p_{T}^{t}$ distribution, etc., while the functions $c_{a b}^{I}(a, b=r, k, n)$ induce P- and CP-even $t \bar{t}$ spin correlations.

ii) The functions $b_{r}^{ \pm}, b_{k}^{ \pm}$are generated by P-violating interactions and induce longitudinal $t$ and $\bar{t}$ polarizations (i.e., a polarization in the production plane). Non-zero differences $b_{r}^{+}-b_{r}^{-}, b_{k}^{+}-b_{k}^{-}$, which are $\mathrm{P}-, \mathrm{CP}-$, and $\mathrm{CPT}_{\mathrm{N}^{-}}$odd, require $\mathrm{CP}$ violation and absorptive parts. 
iii) The coefficient functions $b_{n}^{ \pm}$are generated by $\mathrm{P}$ - and CP-even absorptive parts in the scattering amplitudes, which is the case at 1-loop in the SM, and already at tree level by P-invariant, but CP-violating (effective) interactions. If interactions of the latter type are present then $b_{n}^{+}-b_{n}^{-} \neq 0$. The functions $b_{n}^{ \pm}$induce a transverse $t$ and $\bar{t}$ polarization (i.e., a polarization normal to the production plane), which would differ for $t$ and $\bar{t}$ if non-standard CP-violating interactions are present.

iv) Non-zero coefficient functions $c_{k n}, c_{r n}$ require P-odd but CP-even absorptive contributions to the scattering amplitudes. In the SM they are generated by the absorptive parts of the 1-loop mixed QCD-weak corrections, and they are very small, see section 4.

v) The coefficient functions $c_{r}, c_{k}$ are generated by $\mathrm{P}$ - and CP-violating interactions. As is well known, in the SM CP violating effects in flavor-diagonal reactions like $a b \rightarrow t \bar{t}$ generated by the Kobayashi-Maskawa phase are tiny. Observable CP effects in hadronic $t \bar{t}$ production requires sizeable NP CP violation.

vi) A non-zero coefficient function $c_{n}$ requires $\mathrm{P}$-even but $\mathrm{CP}$-odd absorptive contributions to the scattering amplitudes. In the SM this coefficient function is tiny.

As an aside we recall that in a time-reversal invariant theory, expectation values of $\mathrm{T}_{\mathrm{N}}$-odd observables can be generated by the absorptive part $i \mathcal{A}_{f i}=\mathcal{T}_{f i}-\mathcal{T}_{i f}^{*}$ of the $\mathrm{T}$ matrix element $\mathcal{T}_{f i}$ of a reaction $i \rightarrow f$. T-invariance and the unitarity of the $S$ matrix imply

$$
\left|\mathcal{T}_{f i}\right|^{2}-\left|\mathcal{T}_{\hat{f} \hat{i}}\right|^{2}=-2 \operatorname{Im}\left(\mathcal{A}_{f i} \mathcal{T}_{f i}^{*}\right)-\left|\mathcal{A}_{f i}\right|^{2}
$$

where $\hat{i}, \hat{f}$ denote the initial and final state with 3 -momenta and spins reversed. The left-hand side of this equation has the generic form of a $\mathrm{T}_{\mathrm{N}}$-odd observable.

\section{Effective NP Lagrangian for hadronic top quark pair production}

We assume that new physics (NP) effects in hadronic $t \bar{t}$ production and decay are induced by new heavy particle exchanges, characterized by a mass scale $\Lambda$. Then one may construct a local effective Lagrangian $\mathcal{L}_{\mathrm{NP}}$ in terms of the $\mathrm{SM}$ degrees of freedom which respects the SM (gauge) symmetries and describes possible non-resonant new physics interaction structures as long as the moduli of the kinematic invariants of the $t \bar{t}$ production and decay processes $\lesssim \Lambda$. Recent analyses in the context of $t \bar{t}$ production include [18-22]. Here, we follow [22], but take also CP-violating effective interactions into account.

\section{$3.1 t \bar{t}$ production}

We concentrate on new interactions described by operators of mass-dimension $\operatorname{dim} \mathcal{O} \leq 6$ which manifest themselves in hadronic $t \bar{t}$ production. We consider only that set of operators which contribute to $t \bar{t}$ production through interference with the dominant SM amplitudes, i.e., the QCD tree-level amplitudes. 
Let's first list the operators which involve gluon fields. We do not consider operators that affect only the pure gluon vertices, such as $G G G$ or $G G \tilde{G}$, where $G(\tilde{G})$ denotes the (dual) gluon field strength tensor. For a recent bound on the anomalous coupling associated with $G G G$, see [70]. The strength of $G G \tilde{G}$ is severely constrained by the upper bound on the electric dipole moment of the neutron. For $\operatorname{dim} \mathcal{O} \leq 6$ one then remains with the following three gauge-invariant operators which involve $t, \bar{t}$ and up to three gluon fields $[18,20,22]$ :

$$
\begin{aligned}
\mathcal{O}_{g t} & =\left[\bar{t}_{R} \gamma^{\mu} T^{a} D^{\nu} t_{R}\right] G_{\mu \nu}^{a}, \\
\mathcal{O}_{g Q} & =\left[\bar{Q}_{L} \gamma^{\mu} T^{a} D^{\nu} Q_{L}\right] G_{\mu \nu}^{a}, \\
\mathcal{O}_{\mathrm{CDM}} & =\left[\left(\tilde{\Phi} \bar{Q}_{L}\right) \sigma^{\mu \nu} T^{a} t_{R}\right] G_{\mu \nu}^{a},
\end{aligned}
$$

Here $Q_{L}=\left(t_{L}, b_{L}\right)$ is the left-handed third generation doublet and $\tilde{\Phi}=i \sigma_{2} \Phi^{\dagger}=\left(\phi_{0}^{*},-\phi_{-}\right)$ is the charge-conjugate Higgs doublet field. Moreover, $D_{\mu}=\partial_{\mu}+i g_{s} T^{a} G_{\mu}^{a}$ and $G_{\mu \nu}^{a}=$ $\partial_{\mu} G_{\nu}^{a}-\partial_{\nu} G_{\mu}^{a}-g_{s} f^{a b c} G_{\mu}^{b} G_{\nu}^{c}$ is the gluon field strength tensor. Furthermore, $T^{a}$ are the generators of $\mathrm{SU}(3)_{c}$ in the fundamental representation, with $\operatorname{tr}\left(T^{a} T^{b}\right)=\delta_{a b} / 2$.

Using the equation of motion for the gluons one can show [22] that the sums $\mathcal{O}_{g t}+\mathcal{O}_{g t}^{\dagger}$ and $\mathcal{O}_{g Q}+\mathcal{O}_{g Q}^{\dagger}$ are given by linear combinations of 4-quark operators given below, and are thus redundant if one uses the 4-quark operators below as independent set. On the other hand, the combinations $\mathcal{O}_{g t}-\mathcal{O}_{g t}^{\dagger}$ and $\mathcal{O}_{g Q}-\mathcal{O}_{g Q}^{\dagger}$ induce CP-violating interactions, which cannot be expressed in terms of the the 4-quark operators below. One can therefore use a hermitean effective Lagrangian with these two operator combinations and with (3.3) that contains three independent complex coefficients. After spontaneous symmetry breaking, $\langle\Phi\rangle=v / \sqrt{2},(v \simeq 246 \mathrm{GeV})$ and restriction to the top-quark gluon sector, one obtains, using operators with definite $\mathrm{P}$ and $\mathrm{CP}$ properties, real and dimensionless coupling parameters, and the (on-shell) top-quark mass $m_{t}$ for setting the mass scale: ${ }^{3}$

$$
\mathcal{L}_{\mathrm{NP}, \mathrm{g}}=-\frac{g_{s}}{2 m_{t}}\left[\hat{\mu}_{t} \bar{t} \sigma^{\mu \nu} T^{a} t G_{\mu \nu}^{a}+\hat{d}_{t} \bar{t} i \sigma^{\mu \nu} \gamma_{5} T^{a} t G_{\mu \nu}^{a}\right]+\frac{g_{s}^{2}}{m_{t}^{2}}\left[\hat{c}_{(--)} \mathcal{O}_{(--)}^{g}+\hat{c}_{(-+)} \mathcal{O}_{(-+)}^{g}\right]
$$

where

$$
\mathcal{O}_{(--)}^{g}=\mathcal{O}_{-}+\mathcal{O}_{-}^{\dagger}, \quad \mathcal{O}_{(-+)}^{g}=i\left(\mathcal{O}_{+}-\mathcal{O}_{+}^{\dagger}\right)
$$

and

$$
\mathcal{O}_{+}=\left[\bar{t} \gamma^{\mu} T^{a} D^{\nu} t\right] G_{\mu \nu}^{a}, \quad \mathcal{O}_{-}=i\left[\bar{t} \gamma^{\mu} \gamma_{5} T^{a} D^{\nu} t\right] G_{\mu \nu}^{a} .
$$

Here $t$ denotes the top-quark Dirac field. In (3.4) the real, dimensionless parameters $\hat{\mu}_{t}$ and $\hat{d}_{t}$ denote the anomalous chromo-magnetic (CMDM) and chromo-electric (CEDM) dipole moments of the top quark in the convention of [15]. Contrary to our previous analysis in [15] we stay here strictly within the effective field theory approach - i.e., we do not interpret these moments as form factors which may have an absorptive part. ${ }^{4}$ The chromo-magnetic

\footnotetext{
${ }^{3}$ Our sign convention for the QCD quark gluon interaction is $\mathcal{L}_{q g}=-g_{s} \bar{q} \gamma^{\mu} T^{a} q G_{\mu}^{a}$.

${ }^{4}$ In [22] only the chromo-magnetic dipole operator was considered. Their parameter $c_{h g}$ is related to $\hat{\mu}_{t}$ by $v \operatorname{Re} c_{h g} /\left(\sqrt{2} \Lambda^{2}\right)=-g_{s} \hat{\mu}_{t} /\left(2 m_{t}\right)$.
} 
dipole operator is $\mathrm{P}$ - and $\mathrm{CP}$-even, $\mathcal{O}_{(-+)}^{g}$ is $\mathrm{P}$-even and $\mathrm{CP}$-odd, while the chromo-electric dipole operator and $\mathcal{O}_{(--)}^{g}$ are both $\mathrm{P}$ - and $\mathrm{CP}$-odd.

Next, we list the set of gauge-invariant $\operatorname{dim} \mathcal{O}=6$ four-quark operators that yield non-zero tree-level interference terms with the QCD amplitude for $q \bar{q} \rightarrow t \bar{t}$. We assume universality of the new interactions with respect to the light quarks $q \neq t$. Because the respective parton distribution functions suppress $t \bar{t}$ production by $q \bar{q}$ annihilation for $q=$ $s, c, b$ as compared to $q=u, d$, we take into account only the contributions with $u, d$ in the initial state.

One is then left with the following 7 gauge invariant operators [20, 22]:

$$
\begin{aligned}
\mathcal{O}_{Q q}^{(8,1)} & =\left(\bar{Q}_{L} \gamma_{\mu} T^{a} Q_{L}\right)\left(\bar{q}_{L} \gamma^{\mu} T^{a} q_{L}\right), \\
\mathcal{O}_{Q q}^{(8,3)} & =\left(\bar{Q}_{L} \gamma_{\mu} T^{a} \sigma^{A} Q_{L}\right)\left(\bar{q}_{L} \gamma^{\mu} T^{a} \sigma^{A} q_{L}\right), \\
\mathcal{O}_{t u}^{(8)} & =\left(\bar{t}_{R} \gamma_{\mu} T^{a} t_{R}\right)\left(\bar{u}_{R} \gamma^{\mu} T^{a} u_{R}\right) \\
\mathcal{O}_{t d}^{(8)} & =\left(\bar{t}_{R} \gamma_{\mu} T^{a} t_{R}\right)\left(\bar{d}_{R} \gamma^{\mu} T^{a} d_{R}\right) \\
\mathcal{O}_{Q u}^{(8)} & =\left(\bar{Q}_{L} \gamma_{\mu} T^{a} Q_{L}\right)\left(\bar{u}_{R} \gamma^{\mu} T^{a} u_{R}\right) \\
\mathcal{O}_{Q d}^{(8)} & =\left(\bar{Q}_{L} \gamma_{\mu} T^{a} Q_{L}\right)\left(\bar{d}_{R} \gamma^{\mu} T^{a} d_{R}\right) \\
\mathcal{O}_{t q}^{(8)} & =\left(\bar{t}_{R} \gamma_{\mu} T^{a} t_{R}\right)\left(\bar{q}_{L} \gamma^{\mu} T^{a} q_{L}\right)
\end{aligned}
$$

Here $q_{L}=\left(u_{L}, u_{L}\right)$ denotes the first generation quark doublet, and $\sigma^{A}$ denote the Pauli matrices $\left(\operatorname{tr}\left(\sigma^{A} \sigma^{B}\right)=2 \delta_{A B}\right)$.

These operators contribute to the effective NP Lagrangian a term of the form $\sum_{i=1}^{7}\left(c_{i} / \Lambda^{2}\right) \mathcal{O}_{i}$, denoting $\mathcal{O}_{Q q}^{(8,1)}=\mathcal{O}_{1}$, etc. Again, the terms which involve $b$ quarks are not of interest to us here. As pointed out in [22] it is advantageous to combine these seven operators such that one gets four isospin-zero operators with definite $\mathrm{P}$ and $\mathrm{C}$ properties and three isospin-one operators. (In the isospin-one case it is not possible to combine the operators such that they have definite properties with repect to $\mathrm{C}$ and $\mathrm{P}$.) In the following $q=(u, d)$ denotes the isospin doublet. One gets

$$
\mathcal{L}_{\mathrm{NP}, \mathrm{q}}=\mathcal{L}_{\mathrm{NP}, 0}+\mathcal{L}_{\mathrm{NP}, 1},
$$

where the isosoin-zero part is

$$
\mathcal{L}_{\mathrm{NP}, 0}=\frac{g_{s}^{2}}{2 m_{t}^{2}} \sum_{I, J=V, A} \hat{c}_{I J} \mathcal{O}_{I J} .
$$

and

$$
\begin{array}{ll}
\mathcal{O}_{V V}=\left(\bar{q} \gamma^{\mu} T^{a} q\right)\left(\bar{t} \gamma_{\mu} T^{a} t\right), & \mathcal{O}_{A A}=\left(\bar{q} \gamma^{\mu} T^{a} \gamma_{5} q\right)\left(\bar{t} \gamma_{\mu} \gamma_{5} T^{a} t\right) \\
\mathcal{O}_{V A}=\left(\bar{q} \gamma^{\mu} T^{a} q\right)\left(\bar{t} \gamma_{\mu} \gamma_{5} T^{a} t\right), & \mathcal{O}_{A V}=\left(\bar{q} \gamma^{\mu} T^{a} \gamma_{5} q\right)\left(\bar{t} \gamma_{\mu} T^{a} t\right)
\end{array}
$$

The isospin-one contribution can be represented in the form

$$
\mathcal{L}_{\mathrm{NP}, 1}=\frac{g_{s}^{2}}{4 m_{t}^{2}} \sum_{i=1}^{3} \hat{c}_{i} \mathcal{O}_{i}^{1},
$$


where

$$
\begin{aligned}
\mathcal{O}_{1}^{1} & =\left(\bar{q} \gamma^{\mu} T^{a} \sigma_{3} q\right)\left(\bar{t} \gamma_{\mu} T^{a} t\right)+\left(\bar{q} \gamma^{\mu} \gamma_{5} T^{a} \sigma_{3} q\right)\left(\bar{t} \gamma_{\mu} T^{a} t\right) \\
\mathcal{O}_{2}^{1} & =\left(\bar{q} \gamma^{\mu} \gamma_{5} T^{a} \sigma_{3} q\right)\left(\bar{t} \gamma_{\mu} \gamma_{5} T^{a} t\right)-\left(\bar{q} \gamma^{\mu} \gamma_{5} T^{a} \sigma_{3} q\right)\left(\bar{t} \gamma_{\mu} T^{a} t\right) \\
\mathcal{O}_{3}^{1} & =\left(\bar{q} \gamma^{\mu} T^{a} \sigma_{3} q\right)\left(\bar{t} \gamma_{\mu} \gamma_{5} T^{a} t\right)+\left(\bar{q} \gamma^{\mu} \gamma_{5} T^{a} \sigma_{3} q\right)\left(\bar{t} \gamma_{\mu} T^{a} t\right)
\end{aligned}
$$

Our isospin-zero and -one couplings $\hat{c}_{I J}, \hat{c}_{i}$ are related to those of [22] by $g_{s}^{2} \hat{c}_{I J} / m_{t}^{2}=c_{I j} / \Lambda^{2}$, $g_{s}^{2} \hat{c}_{1} / m_{t}^{2}=c_{V v}^{\prime} / \Lambda^{2}, g_{s}^{2} \hat{c}_{2} / m_{t}^{2}=c_{A a}^{\prime} / \Lambda^{2}, g_{s}^{2} \hat{c}_{3} / m_{t}^{2}=c_{A v}^{\prime} / \Lambda^{2}$. The relation to the coefficients of the operator basis (3.7)-(3.13) is given in [22]. To summarize, we use

$$
\mathcal{L}_{\mathrm{NP}}=\mathcal{L}_{\mathrm{NP}, \mathrm{g}}+\mathcal{L}_{\mathrm{NP}, \mathrm{q}} \cdot
$$

The NP contributions to the coefficients of the $t \bar{t}$ spin density matrices (2.4) induced by interference with the tree-level QCD amplitudes of $g g, q \bar{q} \rightarrow t \bar{t}$ are listed in appendix A.

A side remark concerns $b \bar{b} \rightarrow t \bar{t}$ which receives, apart from the order $\alpha_{s}^{2}$ QCD term, a SM contribution of order $\alpha_{s} \alpha$ from the interference of the $t$-channel $W$-exchange with possible anomalous $W t b$ couplings - and $s$-channel gluon exchange diagram. These SM terms, which are small, are taken into account in section 4. The contributions from anomalous $W t b$ couplings are neglected, because i) they are empirically known to be small (see the next subsection) and ii) the respective interference terms are suppressed by the $b \bar{b}$ parton luminosity.

\subsection{Top-quark decay}

The SM and NP contributions to the parton matrix elements that describe $t \bar{t}$ production and decay into dileptonic and lepton plus jets final states involve also the top-quark decay density matrices for $t \rightarrow W b \rightarrow \ell \nu b, q \bar{q}^{\prime} b$. The top-decay vertex $t \rightarrow W b$ may also be affected by new physics interactions that can also be parametrized by anomalous couplings (cf., for instance, $[20,61]$ ). We use in the following as spin-analyzers of the (anti)top quark only the charged lepton $\ell^{ \pm}$from $W^{ \pm}$decay, and we consider below only lepton angular correlations and distributions that are inclusive in the lepton energies. It is known [74-77] that these observables are not affected by anomalous couplings from top-quark decay if these couplings are small, i.e., if a linear approximation is justified. This is indeed the case in view of the present upper bounds on the moduli of these couplings that can be inferred from the measured $W$-boson helicity fractions in top-quark decay [78, 79]. In other words, the observables which we analyze in the next section are affected only by possible new physics contributions to $t \bar{t}$ production, which we parametrize by the anomalous couplings discussed above.

We use the results of [80] for the polarized semileptonic and non-leptonic top-quark decays in the SM at NLO QCD.

\subsection{Experimental bounds and domain of applicability}

The size of the anomalous couplings of the NP interactions described by (3.22) are constrained by the experimental data on hadronic $t \bar{t}$ production which so far show no significant 
sign of new physics. A recent (re)analysis of the contributions of only the chromo-moments $\hat{\mu}_{t}$ and $\hat{d}_{t}$ to the $t \bar{t}$ cross section and confrontation with the Tevatron and LHC measurements yields correlated bounds. In terms of our (sign) conventions chosen in (3.4), ref. [50] determined the allowed region $(68 \% \mathrm{CL})$ in the $\hat{\mu}_{t}, \hat{d}_{t}$ plane which may be represented by the bounds $-0.03 \leq \hat{\mu}_{t} \leq 0.02$ and $\left|\hat{d}_{t}\right| \leq 0.15$. Ref. [55] found a similar result: $-0.046 \leq \hat{\mu}_{t} \leq 0.024$ and $\left|\hat{d}_{t}\right| \leq 0.17$ (95\% CL). Ref. [68] took into account the QCD corrections to the cross section contribution of $\hat{\mu}_{t}$ and obtained $-0.019 \leq \hat{\mu}_{t} \leq 0.018(95 \%$ CL), putting $\hat{d}_{t}=0$. The CMS experiment analyzed, for dileptonic $t \bar{t}$ events at $7 \mathrm{TeV}$, the distribution of the difference of the leptonic azimuthal angles, and searched for a contribution of a non-zero $\hat{\mu}_{t}$. Using the predictions of [15] for interpreting their data, CMS obtained the bound [9] $-0.043 \leq \hat{\mu}_{t} \leq 0.117$ (95\% CL). Tighter, but indirect bounds on $\hat{d}_{t}$ and $\hat{\mu}_{t}$ were obtained using the experimental upper bound on the neutron electric dipole moment [42] and data on rare $B$ meson decays [42, 81], respectively. (Prospects for correlated bounds on the chromo moments in single top-quark production in the $t W$ mode were recently discussed in [69].)

A global fit to $t \bar{t}$ data using all dimension-six operators that are relevant to hadronic top quark production was recently performed in [70]. The operator set used in this reference contains also the operators contained in (3.22), albeit in a slightly different basis. One may use the correlated bounds of [70] to get order-of-magnitude bounds on the dimensionless couplings of the four-quark operators (3.14), to wit, $\left|\hat{c}_{I J}\right|,\left|\hat{c}_{i}\right| \lesssim 0.4$.

In the next section we will compute the contributions of the NP interactions (3.22) to a number of observables, and we take into account in that section only the NP contributions that are linear in the anomalous couplings. As the anomalous couplings correspond to non-renormalizable effective interactions, their contributions grow in the regime of large energy-momentum transfer to the $t \bar{t}$ system. In other words, the linear approximation can be applied with confidence only for relatively small anomalous couplings. In order to assess the domain of applicability of this approximation we have considered $t \bar{t}$ production at the LHC $(14 \mathrm{TeV})$. We have computed, for the $t \bar{t}$ cross section and for a few spin correlation observables which will be introduced in the next section, the NP contributions to these observables which are linear and quadratic (respectively bilinear) in the anomalous couplings. Following ref. [22] we define the range of applicability of the linear approximation by requiring that the contributions linear in the anomalous couplings are at least twice as large in magnitude as the bilinear ones. Application of this criterion yields $\left|\hat{\mu}_{t}\right|,\left|\hat{d}_{t}\right| \lesssim 0.15$ and $\left|\hat{c}_{I J}\right| \lesssim 0.1$. (We did not consider the contributions of the anomalous couplings $\hat{c}_{i}$ because they are subleading, see the next section.) Thus, as far as the chromo-moments are concerned, the empirical bounds discussed above are essentially within the domain of applicability of the linear approximation.

\section{Observables and results}

In this section we consider $t \bar{t}$ production at the LHC for center-of-mass energies 8,13 , and $14 \mathrm{TeV}$. First we determine the SM contributions and those of $\mathcal{L}_{\mathrm{NP}}$ to the $t \bar{t}$ cross section $\sigma_{t \bar{t}}$ and the $t \bar{t}$ charge asymmetry $A_{\mathrm{C}}$. Then we introduce, for dileptonic and lepton 
plus jets final states, a set of correlation and polarization observables and compute their distributions.

As far as the SM interactions are concerned we compute the four P-even and CP-even correlation observables that will be defined in section 4.2 at NLO QCD (order $\alpha_{s}^{3}$ ) [13] including the weak and mixed QCD-weak corrections of order $\alpha^{2}, \alpha_{s} \alpha, \alpha_{s}^{2} \alpha, \alpha_{s} \alpha^{2}[14,15$, 98-100]. We label these SM contributions by the acronym 'NLOW'. The mixed QCD-QED contributions to these spin correlation observables have not yet been calculated. We expect them to be small, of the order of a few percent of the QCD corrections. The combinations of polarization observables considered in section 4.2 are, apart from one combination, Podd and/or CP-odd. These observables receive no QCD or QED contributions. Only one of these polarization observables, the sum of the transverse $t$ and $\bar{t}$ polarization, receives, at the order of perturbation theory considered here, QCD contributions of order $\alpha_{s}^{3}$, and mixed QCD-weak and mixed QCD-QED contributions of order $\alpha_{s}^{2} \alpha$, which are taken into account. (Contributions of order $\alpha^{2} \alpha_{s}$ to the transverse polarization are very small and are not considered here.) The label 'EW' refers to the electroweak contributions. Our computational set-up is described in [14].

In the SM the total $\sigma_{t \bar{t}}$ cross section is known to NNLO QCD (order $\alpha_{s}^{4}$ ) including soft-gluon resummation at next-to-next-to-leading logarithmic (NNLL) order [72]. The mixed QCD-weak corrections to the cross section (NLOW) were computed in [98-102] and the mixed QCD-QED corrections were determined in [82]. The NLOW corrections to the LHC $t \bar{t}$ cross section are negative, about $\sim-2 \%$ of the LO QCD cross section, while the QCD-QED corrections are positive, about $\sim 1 \%$ according to [82]. The LHC charge asymmetry $A_{\mathrm{C}}$ defined below is computed at NLO QCD, including the mixed QCD-weak and mixed QCD-QED corrections [86]. These mixed EW corrections are known to be important for $A_{\mathrm{C}}$. The computation of $A_{\mathrm{C}}$ to this order of perturbation theory, which is labeled 'NLO+EW', is present state-of-the-art. The Tevatron charge asymmetry was recently computed to NNLO accuracy [89].

Furthermore, we compute the interferences of the NP amplitudes induced by $\mathcal{L}_{\mathrm{NP}}$ and the tree-level QCD amplitudes, but keep only the terms linear in the anomalous couplings. We employ the following input parameters: for the top-quark mass in the on-shell scheme we use $m_{t}=173.34 \mathrm{GeV}$. Moreover, we use $\Gamma_{t}=1.3 \mathrm{GeV}, m_{Z}=91.2 \mathrm{GeV}, m_{W}=$ $80.4 \mathrm{GeV}, \Gamma_{W}=2.09 \mathrm{GeV}, m_{H}=125 \mathrm{GeV}, \alpha\left(m_{t}\right)=0.008$, and we use the CT10 NLO parton distribution functions (PDF) [83]. This PDF set provides also the QCD coupling $\alpha_{s}$ in the $\overline{\mathrm{MS}}$ scheme. For the renormalization and factorization scale, we use $\mu_{\mathrm{R}}=\mu_{\mathrm{F}} \equiv \mu$, $\mu=m_{t}$, and estimate scale uncertainties by varying $\mu$ between $m_{t} / 2$ and $2 m_{t}$.

\subsection{Total $t \bar{t}$ cross section and charge asymmetry at the LHC}

Our results for $\sigma_{t \bar{t}}$ are listed in table 2. The uncertainties refer to the scale uncertainties. In the linear approximation, only the $\mathrm{P}$ - and $\mathrm{CP}$-even operators contained in $\mathcal{L}_{\mathrm{NP}}$ contribute; the CMDM operator, whose contribution is largest, the isospin-zero operator $\mathcal{O}_{V V}$, and the P-even part of the isospin-one operator $\mathcal{O}_{1}^{1}$, whose contribution is smallest. The smallness of the isospin- 1 compared to the isospin- 0 contribution to $\sigma_{t \bar{t}}$, to the charge asymmetry defined below, and to the observables of the next section is due to the fact that a sizeable 


\begin{tabular}{|c|c|c|c|c|c|}
\hline \multicolumn{2}{|c|}{} & NLOW & $\propto \hat{c}_{V V}$ & $\propto \hat{c}_{1}$ & $\propto \hat{\mu}_{t}$ \\
\hline \multirow{5}{*}{$\sigma[\mathrm{pb}]$} & $8 \mathrm{TeV}$ & $207.78_{-25.95}^{+23.51}$ & $246.23_{-67.10}^{+49.08}$ & $32.33_{-8.23}^{+6.13}$ & $761.03_{-178.27}^{+255.18}$ \\
& $13 \mathrm{TeV}$ & $687.32_{-80.23}^{+78.35}$ & $581.16_{-138.98}^{+104.82}$ & $71.06_{-15.90}^{+12.23}$ & $2490.91_{-527.16}^{+723.94}$ \\
& $14 \mathrm{TeV}$ & $814.38_{-94.02}^{+92.89}$ & $654.65_{-153.50}^{+116.20}$ & $79.14_{-17.40}^{+13.37}$ & $2945.65_{-612.79}^{+836.38}$ \\
\hline \multicolumn{2}{|c|}{} & $\mathrm{NLO}+\mathrm{EW}$ & $\propto \hat{c}_{A A}$ & \multicolumn{2}{|c|}{$\propto \hat{c}_{2}$} \\
\hline \multirow{2}{*}{$A_{\mathrm{C}}$} & $8 \mathrm{TeV}$ & $\left(1.11_{-0.04}^{+0.04}\right) \cdot 10^{-2}$ & $0.446_{-0.017}^{+0.017}$ & \multicolumn{2}{|c|}{$\left(9.26_{-0.39}^{+0.42}\right) \cdot 10^{-2}$} \\
& $13 \mathrm{TeV}$ & $\left(0.75_{-0.05}^{+0.04}\right) \cdot 10^{-2}$ & $0.341_{-0.011}^{+0.011}$ & $\left(7.07_{-0.27}^{+0.27}\right) \cdot 10^{-2}$ \\
& $14 \mathrm{TeV}$ & $\left(0.66_{-0.04}^{+0.05}\right) \cdot 10^{-2}$ & $0.327_{-0.010}^{+0.010}$ & $\left(6.77_{-0.25}^{+0.25}\right) \cdot 10^{-2}$ \\
\hline
\end{tabular}

Table 2. The SM and NP contributions to the $t \bar{t}$ cross section and the LHC charge asymmetry (4.1).

fraction of these contributions comes from the kinematic region near threshold. In this region, the contributions of $u \bar{u} \rightarrow t \bar{t}$ and $d \bar{d} \rightarrow t \bar{t}$ are of the same order. The SM result for the cross section listed in table 2 is given here only for reference purposes. Comparing experimental results to predictions one should use the most precise available SM result on $\sigma_{t \bar{t}}$. This is the result of [72] mentioned above. Rather than performing a three-parameter fit to cross section results at the Tevatron and the LHC ( 7 and $8 \mathrm{TeV})$ which is left to a future analysis, we make here a crude estimate of the bound on $\hat{c}_{V V}$ by putting the anomalous couplings $\hat{\mu}_{t}$ and $\hat{c}_{1}$ to zero. Using, for $8 \mathrm{TeV}, \sigma_{\text {exp. }}=241.5 \pm 8.5 \mathrm{pb}$ [84] and the NNLO+NNLL QCD prediction $[72,85] \sigma_{\text {th }}=253 \pm 14 \mathrm{pb}$ and neglecting the electroweak contributions, we get the $95 \%$ C.L. bound $-0.18 \leq \hat{c}_{V V} \leq 0.09$. The lower bound is outside of the condition $\left|\hat{c}_{V V}\right| \lesssim 0.1$ for the linear approximation derived in section 3.3 if the other anomalous couplings are put to zero.

Table 2 contains also our SM and NP prediction for the $t \bar{t}$ charge asymmetry at the LHC which is defined by

$$
A_{\mathrm{C}}=\frac{\sigma(\Delta|y|>0)-\sigma(\Delta|y|<0)}{\sigma(\Delta|y|>0)+\sigma(\Delta|y|<0)}
$$

where $\Delta|y|=\left|y_{t}\right|-\left|y_{\bar{t}}\right|$ is the difference of the moduli of the $t$ and $\bar{t}$ rapidities in the laboratory frame. Our SM predictions, labeled 'NLO + EW', contain apart from the mixed QCD-weak interaction corrections also the mixed QCD-QED corrections of order $\alpha_{s}^{2} \alpha$ [86]. We have computed $A_{\mathrm{C}}$ by expanding the ratio (4.1), see eq. (4.22) below. The NLOW predictions in table 2 are in accord with the results of $[86,87]$ where different PDF sets were used. The NP contributions result from the isospin-zero operator $\mathcal{O}_{A A}$ and the P-even part of the isospin-one operator $\mathcal{O}_{2}^{1}$. These operators induce contributions to the differential cross section that are odd under interchange of the $t$ and $\bar{t}$ momenta while those of the initial (anti)quark are kept fixed. Using the recent CMS measurement [88] with their $8 \mathrm{TeV}$ data, $A_{\mathrm{C}}=0.001 \pm 0.008$, and putting the coupling $\hat{c}_{2}$ to zero, we obtain the $95 \%$ C.L. bound $-0.06 \leq \hat{c}_{A A} \leq 0.01$; cf. also $[88,93]$. This result justifies a posteriori the use of the linear approximation. New physics contributions to the $t \bar{t}$ charge asymmetry $A_{F B}$ at the Tevatron were analyzed in terms of the above effective interactions in [22, 90-92]. The latest experimental results on the Tevatron asymmetry $A_{F B}$ are somewhat larger - 


\begin{tabular}{|c|c|c|}
\hline Observable & no cut & $m_{t \bar{t}}<500 \mathrm{GeV}$ \\
\hline$\delta \sigma_{t \bar{t}}[\mathrm{pb}]$ & 440 & 242 \\
\hline$\delta A_{\mathrm{C}}$ & $4.1 \times 10^{-2}$ & $1.7 \times 10^{-2}$ \\
\hline
\end{tabular}

Table 3. Moduli of maximal differences between future experimental result and SM prediction, for the LHC $(13 \mathrm{TeV})$, for which the linear approximation can be applied.

but compatible - with the most precise SM results [89]. This implies $\hat{c}_{A A}>0$ and $\hat{c}_{A A}$ is bounded from above. The interaction described by $\mathcal{O}_{A A}$ is the effective field theory version of a number of NP models which were invoked to resolve the seeming discrepancy between the experimental results on the Tevatron $A_{F B}$ and the SM predictions. These models include the axigluon model and flavor-changing $t$-channel $Z^{\prime}$ exchange in $u \bar{u} \rightarrow t \bar{t}$; see [94] for a recent overview of the NP models discussed in the context of the $t \bar{t}$ charge asymmetry.

The linear approximation for the contributions of the NP interactions to the cross section and the charge asymmetry is justified if $\left|\hat{\mu}_{t}\right|<\left|\hat{\mu}_{t, \max }\right| \simeq 0.15$ and $\left|\hat{c}_{I J}\right|<\left|\hat{c}_{I J \text {,max }}\right| \simeq$ 0.1, see section 3.3. We assume in the following that the latter bound holds also for anomalous couplings $\hat{c}_{i}$. With these bounds we may make a crude estimate of the maximal difference between (future) experimental measurements of an observable and the SM prediction that will still allow the the use of the linear approximation. In the case of the $t \bar{t}$ cross section, we estimate this maximal difference by

$$
\delta \sigma_{t \bar{t}}=\left|\sigma_{t \bar{t}}^{\exp .}-\sigma_{t \bar{t}}^{\mathrm{SM}}\right|_{\max }=\left|a_{1} \hat{c}_{V V, \max }\right|+\left|a_{2} \hat{c}_{1, \max }\right|+\left|a_{3} \hat{\mu}_{t, \max }\right|,
$$

where the coefficients $a_{i}$ are given in table 2. (We take the values of the $a_{i}$ for $\mu=m_{t}$.) The maximal difference $\delta A_{\mathrm{C}}$ is determined in analogous fashion. In table 3 our estimates of $\delta \sigma_{t \bar{t}}$ and $\delta A_{\mathrm{C}}$ are given for the LHC $(13 \mathrm{TeV})$. As these are crude estimates we have refrained from taking the scale uncertainties of the $a_{i}$ into account. For the other LHC center-ofmass energies, the $\delta \sigma_{t \bar{t}}$ and $\delta A_{\mathrm{C}}$ are obtained in analogous fashion using the coefficients of table 2 .

Table 4 contains our predictions for $\sigma_{t \bar{t}}$ and $A_{\mathrm{C}}$ for events with $t \bar{t}$ invariant mass $M_{t \bar{t}} \leq 500 \mathrm{GeV}$. The mixed QCD-QED contributions to $\sigma_{t \bar{t}}\left(M_{t \bar{t}} \leq 500 \mathrm{GeV}\right)$, which are not included in this table, add $\sim 1 \%$ to the cross section values given in this table. The NNLO QCD corrections are not yet available. For $A_{\mathrm{C}}$, the ratio of the isospin-one and isospinzero NP contributions is approximately the same as in the inclusive case listed in table 2 . In order to determine constraints on both anomalous couplings $\hat{c}_{A A}$ and $\hat{c}_{2}$ from data on the LHC charge asymmetry alone, one may compute the differential charge asymmetry in narrower bins of $M_{t \bar{t}}$, or bins of the rapidity and transverse momentum of the $t \bar{t}$ system.

In analogy to (4.2) one may estimate the maximal differences $\delta \sigma_{t \bar{t}}\left(M_{t \bar{t}} \leq 500 \mathrm{GeV}\right)$ and $\delta A_{\mathrm{C}}\left(M_{t \bar{t}} \leq 500 \mathrm{GeV}\right)$ that would allow the use of the linear approximation. They are given in table 3 .

In the region where, for instance, the $t \bar{t}$ invariant mass $M_{t \bar{t}}$ is large, the linear approximation in the NP couplings can no longer be justified just by assuming that the anomalous couplings are small, see section 3.3. While it is not difficult to compute $\sigma_{t \bar{t}}$ and $A_{\mathrm{C}}$ be- 


\begin{tabular}{|c|c|c|c|c|c|}
\hline \multicolumn{2}{|c|}{-} & NLOW & $\propto \hat{c}_{V V}$ & $\propto \hat{c}_{1}$ & $\propto \hat{\mu}_{t}$ \\
\hline \multirow{4}{*}{$\sigma[\mathrm{pb}]$} & $8 \mathrm{TeV}$ & $129.73_{-16.19}^{+15.81}$ & $104.67_{-25.43}^{+19.16}$ & $12.89_{-2.89}^{+2.22}$ & $480.35_{-108.09}^{+152.01}$ \\
& $13 \mathrm{TeV}$ & $394.22_{-45.95}^{+47.75}$ & $212.03_{-43.75}^{+34.11}$ & $23.98_{-4.58}^{+3.61}$ & $1455.30_{-292.17}^{+393.45}$ \\
& $14 \mathrm{TeV}$ & $461.06_{-53.02}^{+55.77}$ & $234.11_{-47.01}^{+36.95}$ & $26.12_{-4.86}^{+3.88}$ & $1702.52_{-335.37}^{+448.04}$ \\
\hline \multicolumn{2}{|c|}{-} & $\mathrm{NLO}+\mathrm{EW}$ & $\propto \hat{c}_{A A}$ & \multicolumn{2}{|c|}{$\propto \hat{c}_{2}$} \\
\hline \multirow{2}{*}{$A_{\mathrm{C}}$} & $8 \mathrm{TeV}$ & $\left(0.96_{-0.04}^{+0.04}\right) \cdot 10^{-2}$ & $0.209_{-0.011}^{+0.011}$ & $\left(4.38_{-0.24}^{+0.25}\right) \cdot 10^{-2}$ \\
& $13 \mathrm{TeV}$ & $\left(0.63_{-0.04}^{+0.04}\right) \cdot 10^{-2}$ & $0.141_{-0.007}^{+0.007}$ & $\left(2.89_{-0.15}^{+0.16}\right) \cdot 10^{-2}$ \\
& $14 \mathrm{TeV}$ & $\left(0.50_{-0.04}^{+0.04}\right) \cdot 10^{-2}$ & $0.133_{-0.006}^{+0.006}$ & $\left(2.71_{-0.14}^{+0.14}\right) \cdot 10^{-2}$ \\
\hline
\end{tabular}

Table 4. The SM and NP contributions to the $t \bar{t}$ cross section and the LHC charge asymmetry (4.1) events with $M_{t \bar{t}} \leq 500 \mathrm{GeV}$.

yond this approximation, the following complication in the comparison with data arises: there is now a proliferation of contributions (being odd in the scattering angle $y$ defined in section 2) which involve, apart from $\hat{c}_{A A}$ and $\hat{c}_{2}$, also the other anomalous couplings. Information on both $\hat{c}_{A A}$ and $\hat{c}_{2}$ can be obtained either from considering $A_{\mathrm{C}}$ at different c.m. energies and the Tevatron asymmetry simultaneously, or from $A_{\mathrm{C}}$ and (future) measurements of the inclusive $t \bar{t}$ spin correlation and polarization observables defined in the next section.

\subsection{Angular distributions and correlations for dilepton and $\ell+$ jets events}

We analyze $t \bar{t}$ production at the LHC and subsequent decay into dileptonic final states,

$$
p p \rightarrow t+\bar{t}+X \rightarrow \ell^{+} \ell^{-}+\text {jets }+E_{T}^{\text {miss }},
$$

and into lepton plus jets final states,

$$
\begin{aligned}
& p p \rightarrow t+\bar{t}+X \rightarrow \ell^{+}+\text {jets }+E_{T}^{\text {miss }}, \\
& p p \rightarrow t+\bar{t}+X \rightarrow \ell^{-}+\text {jets }+E_{T}^{\text {miss }},
\end{aligned}
$$

where $\ell, \ell^{\prime}=e, \mu, \tau$.

For the dileptonic events (4.3) we consider four-fold dilepton angular distributions. Without acceptance cuts, besides cuts on the $t \bar{t}$ invariant mass, these normalized distributions have the form

$$
\frac{1}{\sigma} \frac{d \sigma}{d \Omega_{+} d \Omega_{-}}=\frac{1}{(4 \pi)^{2}}\left(1+\mathbf{B}_{1}^{\prime} \cdot \hat{\ell}_{+}+\mathbf{B}_{2}^{\prime} \cdot \hat{\ell}_{-}-\hat{\ell}_{+} \cdot C^{\prime} \cdot \hat{\ell}_{-}\right)
$$

where $d \Omega=d \cos \theta d \phi$. The unit vectors $\hat{\ell}_{+}, \hat{\ell}_{-}$are the $\ell^{+}$and $\ell^{\prime-}$ directions of flight in the $t$ and $\bar{t}$ rest frames, respectively, which are reached from the $t \bar{t}$ ZMF by rotation-free boosts. The coefficients, i.e., the vectors $\mathbf{B}_{1}^{\prime}, \mathbf{B}_{2}^{\prime}$ and the matrix $C^{\prime}$ contain the information from the $t \bar{t}$ production and the $t$ and $\bar{t}$ decay density matrices, which may be computed in the SM or in a NP model. As already mentioned in section 3.2, we consider the main SM 
top-decay mode $t \rightarrow W b$. We parametrize $\hat{\ell}_{+}, \hat{\ell}_{-}$in terms of spherical coordinates, but we allow for different polar axes, i.e., different reference axes $\hat{\mathbf{a}}$ and $\hat{\mathbf{b}}$. We define

$$
\cos \theta_{+}=\hat{\ell}_{+} \cdot \hat{\mathbf{a}}, \quad \cos \theta_{-}=\hat{\ell}_{-} \cdot \hat{\mathbf{b}} .
$$

Integrating over the azimuthal angles we obtain the polar angle double distributions for a choice of reference axes $\hat{\mathbf{a}}, \hat{\mathbf{b}}$ :

$$
\frac{1}{\sigma} \frac{d \sigma}{d \cos \theta_{+} d \cos \theta_{-}}=\frac{1}{4}\left(1+B_{1} \cos \theta_{+}+B_{2} \cos \theta_{-}-C \cos \theta_{+} \cos \theta_{-}\right) .
$$

The sign in front of $C$ is chosen such that in the absence of acceptance cuts $[12,13]$ :

$$
C(\hat{\mathbf{a}}, \hat{\mathbf{b}})=\kappa_{\ell}^{2} \frac{\sigma(\uparrow \uparrow)+\sigma(\downarrow \downarrow)-\sigma(\uparrow \downarrow)-\sigma(\downarrow \uparrow)}{\sigma(\uparrow \uparrow)+\sigma(\downarrow \downarrow)+\sigma(\uparrow \downarrow)+\sigma(\downarrow \uparrow)}
$$

where the ratio on the right-hand side of this formula is the double spin-asymmetry at the level of the $t, \bar{t}$ intermediate states. (The first (second) arrow refers to the spin state of the $t(\bar{t})$ quark with respect to the axis $\hat{\mathbf{a}}(\hat{\mathbf{b}})$.) The top-spin analyzing power of the charged lepton from top decay is denoted by $\kappa_{\ell}$. (In the convention used here, $\kappa_{\ell^{+}}=\kappa_{\ell^{-}}=\kappa_{\ell}$.) At NLO QCD its value is $\kappa_{\ell}=0.999$ [80].

The polarization degrees of the ensembles of $t$ and $\bar{t}$ quarks in $t \bar{t}$ events with respect to the reference axes $\hat{\mathbf{a}}, \hat{\mathbf{b}}$ are given by

$$
P(\hat{\mathbf{a}})=\left\langle 2 \mathbf{S}_{t} \cdot \hat{\mathbf{a}}\right\rangle, \quad \bar{P}(\hat{\mathbf{b}})=\left\langle 2 \mathbf{S}_{\bar{t}} \cdot \hat{\mathbf{b}}\right\rangle,
$$

where $\mathbf{S}_{t}, \mathbf{S}_{\bar{t}}$ denotes the $t$ and $\bar{t}$ spin operator, respectively. They are related to $B_{1,2}$ by

$$
B_{1}(\hat{\mathbf{a}})=P(\hat{\mathbf{a}}) \kappa_{\ell}, \quad B_{2}(\hat{\mathbf{b}})=-\bar{P}(\hat{\mathbf{b}}) \kappa_{\ell} .
$$

That is, the relative signs in front of the $B_{1,2}$ in (4.8) are chosen such that in a CP-invariant theory and for the choice $\hat{\mathbf{a}}=-\hat{\mathbf{b}}$ we have

$$
B_{1}=B_{2} .
$$

Our choice of reference axes $\hat{\mathbf{a}}$ and $\hat{\mathbf{b}}$ which we use here is adapted to the orthonormal basis at the parton level introduced in section 2, but is not identical to it. As in section 2, we use the unit vector $\hat{\mathbf{k}}$ which is the top quark direction of flight in the $t \bar{t}$ ZMF. Moreover, we use the direction of one of the proton beams in the laboratory frame, $\hat{\mathbf{p}}_{p}$, and define unit vectors $\hat{\mathbf{r}}_{p}$ and $\hat{\mathbf{n}}_{p}$ as follows:

$$
\begin{array}{rlrl}
\hat{\mathbf{p}}_{p}=(0,0,1), & \hat{\mathbf{r}}_{p}=\frac{1}{r_{p}}\left(\hat{\mathbf{p}}_{p}-y_{p} \hat{\mathbf{k}}\right), & \hat{\mathbf{n}}_{p}=\frac{1}{r_{p}}\left(\hat{\mathbf{p}}_{p} \times \hat{\mathbf{k}}\right), \\
y_{p}=\hat{\mathbf{p}}_{p} \cdot \hat{\mathbf{k}}, & r_{p}=\sqrt{1-y_{p}^{2}} .
\end{array}
$$

Only in the case of $2 \rightarrow 2$ parton reactions and if the incoming parton 1 is parallel to $\hat{\mathbf{p}}_{p}$, the unit vectors defined in (2.5) are the same as those in (4.13). With the set (4.13) 


\begin{tabular}{|cc|c|c|}
\hline & label & $\hat{\mathbf{a}}$ & $\hat{\mathbf{b}}$ \\
\hline transverse & $\mathrm{n}$ & $\operatorname{sign}\left(y_{p}\right) \hat{\mathbf{n}}_{p}$ & $-\operatorname{sign}\left(y_{p}\right) \hat{\mathbf{n}}_{p}$ \\
r axis & $\mathrm{r}$ & $\operatorname{sign}\left(y_{p}\right) \hat{\mathbf{r}}_{p}$ & $-\operatorname{sign}\left(y_{p}\right) \hat{\mathbf{r}}_{p}$ \\
helicity & $\mathrm{k}$ & $\hat{\mathbf{k}}$ & $-\hat{\mathbf{k}}$ \\
\hline
\end{tabular}

Table 5. Choice of reference axes. The unit vectors $\hat{\mathbf{n}}_{p}, \hat{\mathbf{r}}_{p}$ and the variable $y_{p}$ are defined in (4.13).

we define the reference axes $\hat{\mathbf{a}}$ and $\hat{\mathbf{b}}$ listed in table 5 . The factors $\operatorname{sign}\left(y_{p}\right)$ are required because of the Bose symmetry of the initial $g g$ state.

Instead of extracting the spin correlation coefficients $C$ (for a choice of axes) from the double distributions (4.8) one may analyze the 1-dimensional distributions of the observables

$$
\xi=\cos \theta_{+} \cos \theta_{-} .
$$

Labels are suppressed here, i.e., we consider in the following $\xi_{n n}, \xi_{r r}, \xi_{k k}, \xi_{r k}, \xi_{r n}$, etc. The one-dimensional distribution is obtained in standard fashion by inserting

$$
1=\int d \xi \delta\left(\xi-\cos \theta_{+} \cos \theta_{-}\right)
$$

into the integral $\int d \cos \theta_{+} d \cos \theta_{-}$over the double distribution (4.8). One arrives at

$$
\frac{1}{\sigma} \frac{d \sigma}{d \xi}=\frac{1}{2}(1-C \xi) \ln \left(\frac{1}{|\xi|}\right)
$$

which may be used for fits to unfolded experimental results. We recall that

$$
C=-9\langle\xi\rangle \text {. }
$$

In this paper we apply the distributions (4.8) and (4.16) only to dileptonic final states. Integrating out one of the cosines in the double distribution (4.8) one gets

$$
\frac{1}{\sigma} \frac{d \sigma}{d \cos \theta_{ \pm}}=\frac{1}{2}\left(1+B_{1,2} \cos \theta_{ \pm}\right)
$$

The slope of this distribution, $B_{1}\left(B_{2}\right)$, measures the polarization of $t(\bar{t})$ quarks with respect to the chosen reference axis. One may also compute/measure these polarizations using the relation $B_{1,2}=3\left\langle\cos \theta_{ \pm}\right\rangle$. If no acceptance cuts are applied, the coefficient $B_{1}\left(B_{2}\right)$ is the same both for the distribution of $\ell^{+}\left(\ell^{-}\right)$from dilepton and from lepton plus jets events.

From now on we use the following notation. The label $(a, b)$ refers to the choice of reference axes $\hat{\mathbf{a}}$ and $\hat{\mathbf{b}}$ from table 5 . The correlation coefficient $C(a, b)$ in (4.8) or (4.16) is associated with this choice of axes, and likewise $B_{1}(a)$ and $B_{2}(b)$.

In addition to (4.18), we compute also the top and antitop polarization with respect to another set of reference axes:

$$
B_{1}\left(k^{*}\right), \quad B_{2}\left(k^{*}\right), \quad B_{1}\left(r^{*}\right), \quad B_{2}\left(r^{*}\right)
$$


where the labels $k^{*}$ and $r^{*}$ refer to the following vectors:

$$
\begin{array}{lll}
k^{*}: & \hat{\mathbf{a}}=\operatorname{sign}(\Delta|y|) \hat{\mathbf{k}}, & \hat{\mathbf{b}}=-\operatorname{sign}(\Delta|y|) \hat{\mathbf{k}}, \\
r^{*}: & \hat{\mathbf{a}}=\operatorname{sign}(\Delta|y|) \operatorname{sign}\left(y_{p}\right) \hat{\mathbf{r}}_{p}, & \hat{\mathbf{b}}=-\operatorname{sign}(\Delta|y|) \operatorname{sign}\left(y_{p}\right) \hat{\mathbf{r}}_{p} .
\end{array}
$$

Here $\Delta|y|=\left|y_{t}\right|-\left|y_{\bar{t}}\right|$ denotes the difference of the moduli of the $t$ and $\bar{t}$ rapidities in the laboratory frame. The reason for introducing these additional polarization observables is that they are sensitive to the NP contributions from the operator $\mathcal{O}_{A V}$ and from a $\mathrm{P}-$ odd combination of the operators $\mathcal{O}_{i}^{1}$ (defined in (3.16) and (3.19), respectively), while $B_{1,2}(k), B_{1,2}(r)$ project onto the contributions of $\mathcal{O}_{V A}$ and $\mathcal{O}_{3}^{1}$.

The coefficients $C(a, b), B_{1}(a)$, and $B_{2}(b)$ (and sums and differences) are listed in table 6. They can be measured with the 2-dimensional distributions (4.8) or with the 1dimensional distributions of $\xi_{a b}$, respectively with (4.18). Column 2 of this table indicates which coefficient of the $i j \rightarrow t \bar{t} X$ production density matrices introduced in section 2 contributes to the respective correlation coefficient. The type of interactions, i.e., the type of contributions in the differential parton cross sections to which a correlation is sensitive, is given in column 3 .

Tables 7, 8 contain our results for the inclusive correlation coefficients $C(a, b), B_{1}(a)$, $B_{2}(b)$ and $B_{1}\left(a^{*}\right), B_{2}\left(b^{*}\right)$ for the LHC at 8,13 , and $14 \mathrm{TeV}$, while the coefficients in tables 9,10 refer to events with $t \bar{t}$ invariant mass $M_{t \bar{t}} \leq 500 \mathrm{GeV}$. Several comments are in order.

1. All normalized differential distributions, respectively the correlation coefficients are ratios. The $\mathrm{P}$ - and $\mathrm{CP}$-even coefficients $C$ are, in the $\mathrm{SM}$ at NLOW and to first order in the anomalous couplings, schematically of the form

$$
C=\frac{N_{0}+\delta N_{1}+\delta N_{\mathrm{NP}}}{\sigma_{0}+\delta \sigma_{1}+\delta \sigma_{\mathrm{NP}}}
$$

where $N_{0}\left(\delta N_{1}\right)$ and $\sigma_{0}\left(\delta \sigma_{1}\right)$ are the contributions at LO QCD (NLOW). A Taylor expansion at NLO in the SM and anomalous couplings yields

$$
C=\left(1-\frac{\delta \sigma_{1}}{\sigma_{0}}-\frac{\delta \sigma_{\mathrm{NP}}}{\sigma_{0}}\right) \frac{N_{0}}{\sigma_{0}}+\frac{\delta N_{1}}{\sigma_{0}}+\frac{\delta N_{\mathrm{NP}}}{\sigma_{0}}+\mathcal{O}\left(\delta^{2}\right) .
$$

We use this expansion which is in the spirit of perturbation theory. ${ }^{5}$

2. As expected (cf. section 4.1), the tables 7-10 show that for all observables the isospin$1 \mathrm{NP}$ contribution from $\mathcal{L}_{\mathrm{NP}, \mathrm{q}}$ is about one order of magnitude smaller than the isospin-0 contribution.

3. Within the Standard Model $C(r, r)$ is, in the inclusive case, the smallest of the four $\mathrm{P}$ - and CP-even correlations. This can be qualitatively understood with simple treelevel arguments. At the $\mathrm{LHC}(8 \mathrm{TeV})$ the $t$ and $\bar{t}$ quarks in the inclusive $t \bar{t}$ sample

\footnotetext{
${ }^{5}$ Ref. [68] analyzed the CMDM contribution $\hat{\mu}_{t}$ to the helicity correlation $C(k, k)$ including the QCD corrections to this anomalous contribution. It was found that these QCD corrections do not improve the sensitivity to $\hat{\mu}_{t}$.
} 


\begin{tabular}{|c|c|c|}
\hline Correlation & & sensitive to \\
\hline$C(n, n)$ & $c_{n n}^{I}$ & P-, CP-even \\
$C(r, r)$ & $c_{r r}^{I}$ & P-, CP-even \\
$C(k, k)$ & $c_{k k}^{I}$ & P-, CP-even \\
$C(r, k)+C(k, r)$ & $c_{r k}^{I}$ & P-, CP-even \\
$C(n, r)+C(r, n)$ & $c_{r n}^{I}$ & P-odd, CP-even, absorptive \\
$C(n, k)+C(k, n)$ & $c_{k n}^{I}$ & P-odd, CP-even, absorptive \\
$C(r, k)-C(k, r)$ & $c_{n}^{I}$ & P-even, CP-odd, absorptive \\
$C(n, r)-C(r, n)$ & $c_{k}^{I}$ & P-odd, CP-odd \\
$C(n, k)-C(k, n)$ & $-c_{r}^{I}$ & P-odd, CP-odd \\
$B_{1}(n)+B_{2}(n)$ & $b_{n}^{I+}+b_{n}^{I-}$ & P-, CP-even, absorptive \\
$B_{1}(n)-B_{2}(n)$ & $b_{n}^{I+}-b_{n}^{I-}$ & P-even, CP-odd \\
$B_{1}(r)+B_{2}(r)$ & $b_{r}^{I+}+b_{r}^{I-}$ & P-odd, CP-even \\
$B_{1}(r)-B_{2}(r)$ & $b_{r}^{I+}-b_{r}^{I-}$ & P-odd, CP-odd, absorptive \\
$B_{1}(k)+B_{2}(k)$ & $b_{k}^{I+}+b_{k}^{I-}$ & P-odd,CP-even \\
$B_{1}(k)-B_{2}(k)$ & $b_{k}^{I+}-b_{k}^{I-}$ & P-odd, CP-odd, absorptive \\
$B_{1}\left(k^{*}\right)+B_{2}\left(k^{*}\right)$ & $b_{k}^{I+}+b_{k}^{I-}$ & P-odd,CP-even \\
$B_{1}\left(k^{*}\right)-B_{2}\left(k^{*}\right)$ & $b_{k}^{I+}-b_{k}^{I-}$ & P-odd, CP-odd, absorptive \\
$B_{1}\left(r^{*}\right)+B_{2}\left(r^{*}\right)$ & $b_{r}^{I+}+b_{r}^{I-}$ & P-odd, CP-even \\
$B_{1}\left(r^{*}\right)-B_{2}\left(r^{*}\right)$ & $b_{r}^{I+}-b_{r}^{I-}$ & P-odd, CP-odd, absorptive \\
\hline
\end{tabular}

Table 6. The correlation coefficients of (4.8), respectively of (4.17), (4.18) and (4.19), and sums and differences for different choices of reference axes.

are on average only moderately relativistic. At or very close to threshold the $t \bar{t}$ system produced at LO QCD by gluon fusion is in a ${ }^{1} S_{0}$ state. Thus, not too far away from the threshold, the spin configuration of the $t \bar{t}$ with respect to the r-axes $\hat{\mathbf{a}}_{r}$ and $\hat{\mathbf{b}}_{r}$ (which point into opposite directions) is preferentially $\uparrow \uparrow$ or $\downarrow \downarrow$, while for relativistic $t \bar{t}$ the configuration $\uparrow \downarrow$ or $\downarrow \uparrow$ dominates. This statement applies also to the $g g \rightarrow t \bar{t}$ contribution to the helicity correlation $C(k, k)$. The net effect for the inclusive sample is that gluon production contributes positively both to the correlation $C(r, r)$ and $C(k, k)$, but the correlation of the $t$ and $\bar{t}$ spins with respect to the $r$-axes is significantly smaller than their correlation with respect to the helicity axes $\hat{\mathbf{a}}=\hat{\mathbf{k}}$, $\hat{\mathbf{b}}=-\hat{\mathbf{k}}$. The $t, \bar{t}$ produced by $q \bar{q}$ annihilation make a negative contribution both to $C(r, r)$ and $C(k, k)$, and it turns out that the magnitude of the contribution is in 


\begin{tabular}{|c|c|c|c|c|c|}
\hline \multicolumn{2}{|l|}{-} & \multirow{2}{*}{$\frac{\mathrm{NLOW}}{0.332_{-0.002}^{+0.002}}$} & $\propto \hat{c}_{V V}$ & $\propto \hat{c}_{1}$ & \multirow{2}{*}{$\frac{\propto \hat{\mu}_{t}}{1.831_{-0.036}^{+0.038}}$} \\
\hline \multirow{3}{*}{$C(n, n)$} & $8 \mathrm{TeV}$ & & $-0.150_{-0.012}^{+0.010}$ & $\left(-1.76_{-0.17}^{+0.15}\right) \cdot 10^{-2}$ & \\
\hline & $13 \mathrm{TeV}$ & $0.326_{-0.002}^{+0.002}$ & $\left(-7.99_{-0.85}^{+0.80}\right) \cdot 10^{-2}$ & $\left(-8.21_{-1.08}^{+0.99}\right) \cdot 10^{-3}$ & $2.025_{-0.024}^{+0.025}$ \\
\hline & $14 \mathrm{TeV}$ & $0.325_{-0.002}^{+0.002}$ & $\left(-7.21_{-0.81}^{+0.75}\right) \cdot 10^{-2}$ & $\left(-7.20_{-0.99}^{+0.94}\right) \cdot 10^{-3}$ & $2.047_{-0.022}^{+0.022}$ \\
\hline \multirow{3}{*}{$C(r, r)$} & $8 \mathrm{TeV}$ & $0.055_{-0.007}^{+0.009}$ & $-0.934_{-0.037}^{+0.033}$ & $-0.122_{-0.006}^{+0.006}$ & $2.313_{-0.031}^{+0.032}$ \\
\hline & $13 \mathrm{TeV}$ & $0.071_{-0.006}^{+0.008}$ & $-0.697_{-0.027}^{+0.027}$ & $\left(-8.46_{-0.43}^{+0.41}\right) \cdot 10^{-2}$ & $2.475_{-0.019}^{+0.020}$ \\
\hline & $14 \mathrm{TeV}$ & $0.072_{-0.006}^{+0.008}$ & $-0.665_{-0.026}^{+0.025}$ & $\left(-7.99_{-0.40}^{+0.39}\right) \cdot 10^{-2}$ & $2.493_{-0.019}^{+0.019}$ \\
\hline \multirow{3}{*}{$C(k, k)$} & $8 \mathrm{TeV}$ & $0.318_{-0.002}^{+0.003}$ & $-1.570_{-0.053}^{+0.049}$ & $-0.209_{-0.010}^{+0.009}$ & $0.964_{-0.009}^{+0.009}$ \\
\hline & $13 \mathrm{TeV}$ & $0.331_{-0.002}^{+0.002}$ & $-1.218_{-0.040}^{+0.039}$ & $-0.151_{-0.007}^{+0.007}$ & $0.917_{-0.006}^{+0.006}$ \\
\hline & $14 \mathrm{TeV}$ & $0.331_{-0.002}^{+0.002}$ & $-1.169_{-0.038}^{+0.037}$ & $-0.143_{-0.006}^{+0.006}$ & $0.911_{-0.005}^{+0.006}$ \\
\hline \multirow{3}{*}{$C(r, k)+C(k, r)$} & $8 \mathrm{TeV}$ & $-0.226_{-0.004}^{+0.004}$ & $-0.430_{-0.018}^{+0.016}$ & $\left(-5.42_{-0.30}^{+0.28}\right) \cdot 10^{-2}$ & $0.720_{-0.010}^{+0.008}$ \\
\hline & $13 \mathrm{TeV}$ & $-0.206_{-0.002}^{+0.002}$ & $-0.306_{-0.014}^{+0.014}$ & $\left(-3.58_{-0.20}^{+0.20}\right) \cdot 10^{-2}$ & $0.740_{-0.002}^{+0.001}$ \\
\hline & $14 \mathrm{TeV}$ & $-0.204_{-0.002}^{+0.004}$ & $-0.290_{-0.012}^{+0.012}$ & $\left(-3.34_{-0.20}^{+0.18}\right) \cdot 10^{-2}$ & $0.740_{-0.004}^{+0.002}$ \\
\hline \multicolumn{2}{|l|}{-} & \multicolumn{4}{|c|}{ NLOW } \\
\hline \multirow{3}{*}{$C(n, r)+C(r, n)$} & $8 \mathrm{TeV}$ & \multicolumn{4}{|c|}{$\left(1.03_{-0.01}^{+0.01}\right) \cdot 10^{-3}$} \\
\hline & $13 \mathrm{TeV}$ & \multicolumn{4}{|c|}{$\left(1.06_{-0.01}^{+0.01}\right) \cdot 10^{-3}$} \\
\hline & $14 \mathrm{TeV}$ & \multicolumn{4}{|c|}{$\left(1.07_{-0.01}^{+0.01}\right) \cdot 10^{-3}$} \\
\hline \multirow{3}{*}{$C(n, k)+C(k, n)$} & $8 \mathrm{TeV}$ & \multicolumn{4}{|c|}{$\left(2.32_{-0.01}^{+0.01}\right) \cdot 10^{-3}$} \\
\hline & $13 \mathrm{TeV}$ & \multicolumn{4}{|c|}{$\left(2.15_{-.007}^{+0.04}\right) \cdot 10^{-3}$} \\
\hline & $14 \mathrm{TeV}$ & \multicolumn{4}{|c|}{$\left(2.12_{-0.08}^{+0.03}\right) \cdot 10^{-3}$} \\
\hline \multicolumn{2}{|l|}{-} & \multicolumn{2}{|c|}{$\propto \hat{c}_{(--)}$} & \multicolumn{2}{|l|}{$\propto \hat{d}_{t}$} \\
\hline \multirow{3}{*}{$C(n, r)-C(r, n)$} & $8 \mathrm{TeV}$ & \multicolumn{2}{|c|}{$-1.321_{-0.020}^{+0.021}$} & \multicolumn{2}{|c|}{$-3.705_{-0.087}^{+0.084}$} \\
\hline & $13 \mathrm{TeV}$ & \multicolumn{2}{|c|}{$-1.226_{-0.004}^{+0.007}$} & \multicolumn{2}{|c|}{$-4.143_{-0.056}^{+0.053}$} \\
\hline & $14 \mathrm{TeV}$ & \multicolumn{2}{|c|}{$-1.217_{-0.002}^{+0.005}$} & \multicolumn{2}{|c|}{$-4.195_{-0.052}^{+0.052}$} \\
\hline \multirow{3}{*}{$C(n, k)-C(k, n)$} & $8 \mathrm{TeV}$ & \multicolumn{2}{|c|}{$-1.665_{-0.058}^{+0.073}$} & \multicolumn{2}{|c|}{$-0.721_{-0.019}^{+0.021}$} \\
\hline & $13 \mathrm{TeV}$ & \multicolumn{2}{|c|}{$-2.157_{-0.087}^{+0.108}$} & $-0.800_{-0 .}^{+0 .}$ & \\
\hline & $14 \mathrm{TeV}$ & -2 & $236_{-0.091}^{+0.112}$ & $-0.805_{-0 .}^{+0 .}$ & \\
\hline
\end{tabular}

Table 7. The correlation coefficients $C$ at NLOW in the SM and the non-zero contributions of the dimensionless anomalous couplings defined in section 3 . The central values correspond to the scales $\mu=m_{t}$. The uncertainties refer to $\mu=m_{t} / 2$ and $\mu=2 m_{t}$.

both cases approximately the same. This is because in this case, at LO QCD, t $\bar{t}$ near threshold is in a ${ }^{3} S_{1}$ state. Thus both with respect to the r-axes and the helicity axes, the spin configuration $\uparrow \downarrow$ or $\downarrow \uparrow$ dominates, both for non-relativistic and relativistic 


\begin{tabular}{|c|c|c|c|c|}
\hline \multicolumn{2}{|l|}{-} & NLOW & $\propto \hat{c}_{V A}$ & $\propto \hat{c}_{3}$ \\
\hline \multirow{3}{*}{$B_{1}(r)+B_{2}(r)$} & $8 \mathrm{TeV}$ & $\left(2.9_{-1.4}^{+1.9}\right) \cdot 10^{-3}$ & $0.298_{-0.014}^{+0.014}$ & $\left(3.89_{-0.23}^{+0.24}\right) \cdot 10^{-2}$ \\
\hline & $13 \mathrm{TeV}$ & $\left(3.2_{-1.7}^{+2.3}\right) \cdot 10^{-3}$ & $0.210_{-0.009}^{+0.009}$ & $\left(2.55_{-0.14}^{+0.14}\right) \cdot 10^{-2}$ \\
\hline & $14 \mathrm{TeV}$ & $\left(3.3_{-1.8}^{+2.3}\right) \cdot 10^{-3}$ & $0.199_{-0.008}^{+0.008}$ & $\left(2.39_{-0.13}^{+0.13}\right) \cdot 10^{-2}$ \\
\hline \multirow{3}{*}{$B_{1}(k)+B_{2}(k)$} & $8 \mathrm{TeV}$ & $\left(6.1_{-1.7}^{+2.3}\right) \cdot 10^{-3}$ & $2.100_{-0.078}^{+0.081}$ & $0.283_{-0.014}^{+0.015}$ \\
\hline & $13 \mathrm{TeV}$ & $\left(8.0_{-2.4}^{+3.4}\right) \cdot 10^{-3}$ & $1.607_{-0.052}^{+0.051}$ & $0.201_{-0.009}^{+0.009}$ \\
\hline & $14 \mathrm{TeV}$ & $\left(8.3_{-3.5}^{+3.5}\right) \cdot 10^{-3}$ & $1.542_{-0.047}^{+0.047}$ & $0.191_{-0.008}^{+0.008}$ \\
\hline \multicolumn{2}{|l|}{-} & NLOW & $\propto \hat{c}_{A V}$ & $\propto \hat{c}_{1}-\hat{c}_{2}+\hat{c}_{3}$ \\
\hline \multirow{3}{*}{$B_{1}\left(r^{*}\right)+B_{2}\left(r^{*}\right)$} & $8 \mathrm{TeV}$ & $<10^{-3}$ & $1.166_{-0.060}^{+0.063}$ & $0.244_{-0.014}^{+0.014}$ \\
\hline & $13 \mathrm{TeV}$ & $<10^{-3}$ & $0.792_{-0.039}^{+0.040}$ & $0.164_{-0.009}^{+0.009}$ \\
\hline & $14 \mathrm{TeV}$ & $<10^{-3}$ & $0.746_{-0.036}^{+0.037}$ & $0.154_{-0.008}^{+0.008}$ \\
\hline \multirow{3}{*}{$B_{1}\left(k^{*}\right)+B_{2}\left(k^{*}\right)$} & $8 \mathrm{TeV}$ & $<10^{-3}$ & $1.204_{-0.052}^{+0.054}$ & $0.251_{-0.012}^{+0.013}$ \\
\hline & $13 \mathrm{TeV}$ & $<10^{-3}$ & $0.883_{-0.034}^{+0.034}$ & $0.183_{-0.008}^{+0.008}$ \\
\hline & $14 \mathrm{TeV}$ & $<10^{-3}$ & $0.842_{-0.032}^{+0.032}$ & $0.174_{-0.007}^{+0.007}$ \\
\hline \multicolumn{2}{|l|}{-} & NLO QCD & \multicolumn{2}{|r|}{ EW } \\
\hline \multirow{3}{*}{$B_{1}(n)+B_{2}(n)$} & $8 \mathrm{TeV}$ & $\left(6.93_{-0.72}^{+0.84}\right) \cdot 10^{-3}$ & \multicolumn{2}{|c|}{$\left(3.44_{-0.07}^{+0.09}\right) \cdot 10^{-3}$} \\
\hline & $13 \mathrm{TeV}$ & $\left(7.45_{-0.72}^{+0.90}\right) \cdot 10^{-3}$ & \multicolumn{2}{|c|}{$\left(3.89_{-0.06}^{+0.06}\right) \cdot 10^{-3}$} \\
\hline & $14 \mathrm{TeV}$ & $\left(7.52_{-0.73}^{+0.90}\right) \cdot 10^{-3}$ & \multicolumn{2}{|c|}{$\left(3.94_{-0.05}^{+0.06}\right) \cdot 10^{-3}$} \\
\hline \multicolumn{2}{|l|}{-} & \multicolumn{3}{|c|}{$\propto \hat{c}_{(-+)}$} \\
\hline \multirow{3}{*}{$B_{1}(n)-B_{2}(n)$} & $8 \mathrm{TeV}$ & \multicolumn{3}{|c|}{$4.148_{-0.128}^{+0.115}$} \\
\hline & $13 \mathrm{TeV}$ & \multicolumn{3}{|c|}{$4.876_{-0.124}^{+0.108}$} \\
\hline & $14 \mathrm{TeV}$ & \multicolumn{3}{|c|}{$4.975_{-0.124}^{+0.109}$} \\
\hline
\end{tabular}

Table 8. The correlation coefficients $B$ at NLOW in the SM and the non-zero contributions of the dimensionless anomalous couplings defined in section 3 . The central values correspond to the scales $\mu=m_{t}$. The uncertainties refer to $\mu=m_{t} / 2$ and $\mu=2 m_{t}$.

$t, \bar{t}$. As a result, the contributions from $g g$ and $q \bar{q}$ initial states almost cancel in the case of $C(r, r)$, while in the case of $C(k, k)$ the positive contribution from the $g g$ channel is significantly larger in magnitude than the negative one from $q \bar{q} \rightarrow t \bar{t}$.

This line of arguments suggests that the ratio of $C(r, r)$ and $C(k, k)$ becomes larger if the $t \bar{t}$ sample is restricted to events not too far away from the $t \bar{t}$ threshold. This is corroborated by the numbers of table 9 . The fact that the correlation $C(n, n)$ 


\begin{tabular}{|c|c|c|c|c|c|}
\hline \multicolumn{2}{|l|}{-} & NLOW & $\propto \hat{c}_{V V}$ & $\propto \hat{c}_{1}$ & $\propto \hat{\mu}_{t}$ \\
\hline \multirow{3}{*}{$C(n, n)$} & $8 \mathrm{TeV}$ & $0.396_{-0.002}^{+0.002}$ & $-0.317_{-0.013}^{+0.012}$ & $\left(-3.89_{-0.22}^{+0.20}\right) \cdot 10^{-2}$ & $1.864_{-0.034}^{+0.035}$ \\
\hline & $13 \mathrm{TeV}$ & $0.403_{-0.002}^{+0.002}$ & $-0.230_{-0.010}^{+0.009}$ & $\left(-2.59_{-0.14}^{+0.14}\right) \cdot 10^{-2}$ & $2.041_{-0.023}^{+0.024}$ \\
\hline & $14 \mathrm{TeV}$ & $0.403_{-0.002}^{+0.002}$ & $-0.219_{-0.009}^{+0.009}$ & $\left(-2.43_{-0.13}^{+0.13}\right) \cdot 10^{-2}$ & $2.062_{-0.022}^{+0.023}$ \\
\hline \multirow{3}{*}{$C(r, r)$} & $8 \mathrm{TeV}$ & $0.137_{-0.005}^{+0.007}$ & $-0.821_{-0.032}^{+0.030}$ & $-0.101_{-0.005}^{+0.005}$ & $2.360_{-0.028}^{+0.031}$ \\
\hline & $13 \mathrm{TeV}$ & $0.168_{-0.003}^{+0.005}$ & $-0.599_{-0.025}^{+0.024}$ & $\left(-6.78_{-0.36}^{+0.35}\right) \cdot 10^{-2}$ & $2.510_{-0.020}^{+0.020}$ \\
\hline & $14 \mathrm{TeV}$ & $0.171_{-0.003}^{+0.005}$ & $-0.571_{-0.023}^{+0.022}$ & $\left(-6.36_{-0.33}^{+0.33}\right) \cdot 10^{-2}$ & $2.527_{-0.018}^{+0.019}$ \\
\hline \multirow{3}{*}{$C(k, k)$} & $8 \mathrm{TeV}$ & $0.413_{-0.003}^{+0.003}$ & $-1.060_{-0.040}^{+0.038}$ & $-0.131_{-0.007}^{+0.006}$ & $0.928_{-0.013}^{+0.010}$ \\
\hline & $13 \mathrm{TeV}$ & $0.446_{-0.008}^{+0.007}$ & $-0.777_{-0.031}^{+0.030}$ & $\left(-8.80_{-0.46}^{+0.44}\right) \cdot 10^{-2}$ & $0.863_{-0.010}^{+0.009}$ \\
\hline & $14 \mathrm{TeV}$ & $0.448_{-0.008}^{+0.009}$ & $-0.741_{-0.029}^{+0.028}$ & $\left(-8.28_{-0.43}^{+0.41}\right) \cdot 10^{-2}$ & $0.855_{-0.010}^{+0.008}$ \\
\hline \multirow{3}{*}{$C(r, k)+C(k, r)$} & $8 \mathrm{TeV}$ & $-0.230_{-0.004}^{+0.004}$ & $-0.430_{-0.016}^{+0.016}$ & $\left(-5.30_{-0.28}^{+0.26}\right) \cdot 10^{-2}$ & $0.980_{-0.014}^{+0.014}$ \\
\hline & $13 \mathrm{TeV}$ & $-0.212_{-0.002}^{+0.010}$ & $-0.316_{-0.012}^{+0.012}$ & $\left(-3.56_{-0.18}^{+0.18}\right) \cdot 10^{-2}$ & $1.054_{-0.010}^{+0.010}$ \\
\hline & $14 \mathrm{TeV}$ & $-0.210_{-0.002}^{+0.002}$ & $-0.300_{-0.012}^{+0.012}$ & $\left(-3.36_{-0.16}^{+0.16}\right) \cdot 10^{-2}$ & $1.062_{-0.010}^{+0.010}$ \\
\hline \multicolumn{2}{|l|}{-} & \multicolumn{4}{|c|}{ NLOW } \\
\hline \multirow{3}{*}{$C(n, r)+C(r, n)$} & $8 \mathrm{TeV}$ & \multicolumn{4}{|c|}{$<10^{-3}$} \\
\hline & $13 \mathrm{TeV}$ & \multicolumn{4}{|c|}{$<10^{-3}$} \\
\hline & $14 \mathrm{TeV}$ & \multicolumn{4}{|c|}{$<10^{-3}$} \\
\hline \multirow{3}{*}{$C(n, k)+C(k, n)$} & $8 \mathrm{TeV}$ & \multirow{3}{*}{\multicolumn{4}{|c|}{$\begin{array}{l}\left(2.64_{-0.05}^{+0.04}\right) \cdot 10^{-3} \\
\left(2.87_{-0.03}^{+0.04}\right) \cdot 10^{-3} \\
\left(2.91_{-0.04}^{+0.02}\right) \cdot 10^{-3}\end{array}$}} \\
\hline & $13 \mathrm{TeV}$ & & & & \\
\hline & $14 \mathrm{TeV}$ & & & & \\
\hline \multicolumn{2}{|l|}{-} & \multicolumn{2}{|c|}{$\propto \hat{c}_{(--)}$} & \multicolumn{2}{|l|}{$\propto \hat{d}_{t}$} \\
\hline \multirow{3}{*}{$C(n, r)-C(r, n)$} & $8 \mathrm{TeV}$ & \multirow{3}{*}{\multicolumn{2}{|c|}{$\begin{array}{l}-0.667_{-0.035}^{+0.035} \\
-0.481_{-0.023}^{+0.023} \\
-0.459_{-0.022}^{+0.021} \\
\end{array}$}} & \multicolumn{2}{|c|}{$-3.746_{-0.091}^{+0.086}$} \\
\hline & $13 \mathrm{TeV}$ & & & \multicolumn{2}{|c|}{$-4.208_{-0.062}^{+0.061}$} \\
\hline & $14 \mathrm{TeV}$ & & & $-4.261_{-0.0}^{+0.0}$ & \\
\hline \multirow{3}{*}{$C(n, k)-C(k, n)$} & $8 \mathrm{TeV}$ & \multicolumn{2}{|c|}{$0.719_{-0.035}^{+0.036}$} & \multirow{3}{*}{\multicolumn{2}{|c|}{$\begin{array}{r}-1.098_{-0.034}^{+0.032} \\
-1.274_{-0.023}^{+0.023} \\
-1.294_{-0.022}^{+0.021}\end{array}$}} \\
\hline & $13 \mathrm{TeV}$ & \multirow{2}{*}{\multicolumn{2}{|c|}{$\begin{array}{l}0.908_{-0.022}^{+0.035} \\
0.929_{-0.020}^{+0.020}\end{array}$}} & & \\
\hline & $14 \mathrm{TeV}$ & & & & \\
\hline
\end{tabular}

Table 9. The correlation coefficients $C$ at NLOW in the SM and the non-zero contributions of the dimensionless anomalous couplings defined in section 3 for $M_{t \bar{t}} \leq 500 \mathrm{GeV}$.

is rather large in the SM can also be understood from total angular momentum conservation.

The measurement of these four correlations would provide sufficient information to simultaneously determine or constrain the CMDM $\hat{\mu}_{t}$ and the anomalous fourquark couplings $\hat{c}_{V V}$ and $\hat{c}_{1}$.

As an aside we remark that the NLOW and the CMDM $\left(\hat{\mu}_{t}\right)$ contribution to the helicity correlation $C(k, k)$ at $8 \mathrm{TeV}$ and the NLOW result for $14 \mathrm{TeV}$ listed in table 7 agree with those computed in [15] and in [14], respectively, with a different PDF set. 


\begin{tabular}{|c|c|c|c|c|}
\hline \multicolumn{2}{|l|}{-} & NLOW & $\propto \hat{c}_{V A}$ & $\propto \hat{c}_{3}$ \\
\hline \multirow{3}{*}{$B_{1}(r)+B_{2}(r)$} & $8 \mathrm{TeV}$ & $\left(2.5_{-2.1}^{+2.3}\right) \cdot 10^{-3}$ & $0.196_{-0.010}^{+0.010}$ & $\left(2.43_{-0.16}^{+0.16}\right) \cdot 10^{-2}$ \\
\hline & $13 \mathrm{TeV}$ & $\left(2.9_{-1.9}^{+2.8}\right) \cdot 10^{-3}$ & $0.134_{-0.006}^{+0.006}$ & $\left(1.52_{-0.09}^{+0.09}\right) \cdot 10^{-2}$ \\
\hline & $14 \mathrm{TeV}$ & $\left(2.9_{-2.1}^{+2.9}\right) \cdot 10^{-3}$ & $0.127_{-0.006}^{+0.006}$ & $\left(1.42_{-0.08}^{+0.08}\right) \cdot 10^{-2}$ \\
\hline \multirow{3}{*}{$B_{1}(k)+B_{2}(k)$} & $8 \mathrm{TeV}$ & $\left(2.6_{-1.5}^{+2.0}\right) \cdot 10^{-3}$ & $0.979_{-0.049}^{+0.050}$ & $0.121_{-0.008}^{+0.008}$ \\
\hline & $13 \mathrm{TeV}$ & $\left(3.5_{-2.0}^{+2.7}\right) \cdot 10^{-3}$ & $0.669_{-0.031}^{+0.032}$ & $\left(7.60_{-0.45}^{+0.45}\right) \cdot 10^{-2}$ \\
\hline & $14 \mathrm{TeV}$ & $\left(3.6_{-2.7}^{+2.8}\right) \cdot 10^{-3}$ & $0.632_{-0.029}^{+0.029}$ & $\left(7.09_{-0.41}^{+0.42}\right) \cdot 10^{-2}$ \\
\hline \multicolumn{2}{|l|}{-} & NLOW & $\propto \hat{c}_{A V}$ & $\propto \hat{c}_{1}-\hat{c}_{2}+\hat{c}_{3}$ \\
\hline \multirow{3}{*}{$B_{1}\left(r^{*}\right)+B_{2}\left(r^{*}\right)$} & $8 \mathrm{TeV}$ & $<10^{-3}$ & $0.988_{-0.053}^{+0.055}$ & $0.207_{-0.012}^{+0.012}$ \\
\hline & $13 \mathrm{TeV}$ & $<10^{-3}$ & $0.659_{-0.033}^{+0.034}$ & $0.135_{-0.007}^{+0.008}$ \\
\hline & $14 \mathrm{TeV}$ & $<10^{-3}$ & $0.620_{-0.031}^{+0.031}$ & $0.126_{-0.007}^{+0.007}$ \\
\hline \multirow{3}{*}{$B_{1}\left(k^{*}\right)+B_{2}\left(k^{*}\right)$} & $8 \mathrm{TeV}$ & $<10^{-3}$ & $0.765_{-0.041}^{+0.042}$ & $0.160_{-0.010}^{+0.009}$ \\
\hline & $13 \mathrm{TeV}$ & $<10^{-3}$ & $0.511_{-0.025}^{+0.026}$ & $0.105_{-0.006}^{+0.006}$ \\
\hline & $14 \mathrm{TeV}$ & $<10^{-3}$ & $0.481_{-0.024}^{+0.024}$ & $\left(9.83_{-0.05}^{+0.05}\right) \cdot 10^{-2}$ \\
\hline \multicolumn{2}{|l|}{-} & NLO QCD & \multicolumn{2}{|c|}{ EW } \\
\hline \multirow{3}{*}{$B_{1}(n)+B_{2}(n)$} & $8 \mathrm{TeV}$ & $\left(4.12_{-0.36}^{+0.44}\right) \cdot 10^{-3}$ & \multirow{3}{*}{\multicolumn{2}{|c|}{$\begin{array}{l}\left(3.14_{-0.05}^{+0.09}\right) \cdot 10^{-3} \\
\left(3.50_{-0.03}^{+0.07}\right) \cdot 10^{-3} \\
\left(3.55_{-0.03}^{+0.05}\right) \cdot 10^{-3}\end{array}$}} \\
\hline & $13 \mathrm{TeV}$ & $\left(4.16_{-0.37}^{+0.45}\right) \cdot 10^{-3}$ & & \\
\hline & $14 \mathrm{TeV}$ & $\left(4.16_{-0.37}^{+0.45}\right) \cdot 10^{-3}$ & & \\
\hline \multicolumn{2}{|l|}{-} & \multicolumn{3}{|c|}{$\propto \hat{c}_{(-+)}$} \\
\hline \multirow{3}{*}{$B_{1}(n)-B_{2}(n)$} & $8 \mathrm{TeV}$ & \multicolumn{3}{|c|}{$2.499_{-0.051}^{+0.047}$} \\
\hline & $13 \mathrm{TeV}$ & \multicolumn{3}{|c|}{$2.754_{-0.038}^{+0.036}$} \\
\hline & $14 \mathrm{TeV}$ & \multicolumn{3}{|c|}{$2.785_{-0.036}^{+0.034}$} \\
\hline
\end{tabular}

Table 10. The correlation coefficients $B$ at NLOW in the SM and the non-zero contributions of the dimensionless anomalous couplings defined in section 3 for $M_{t \bar{t}} \leq 500 \mathrm{GeV}$.

4. The opening angle distribution, $\sigma^{-1} d \sigma / d \cos \varphi=(1-D \cos \varphi) / 2[13]$, which is also a useful spin correlation observable, can be obtained from the above diagonal correlation coefficients. Because the reference axes defined in table 5 form orthonormal bases, we have [13]

$$
D=-\frac{1}{3}[C(n, n)+C(r, r)+C(k, k)] .
$$

This relation can be applied both to the SM and the NP contributions given in the tables below. The correlation coefficients $D$ computed with (4.23) agree with those computed in [15] and [14] for $8 \mathrm{TeV}$ and $14 \mathrm{TeV}$, respectively.

5. One may also consider the spin correlations $C\left(r^{*}, k\right), C\left(k, r^{*}\right)$, and $C\left(k, k^{*}\right)$, where the $k^{*}$ and $r^{*}$ axes are defined in (4.20) and (4.21). These observables are spin 
correlation analogues of the charge asymmetry $A_{\mathrm{C}}$. They receive contributions from the entries of the $t \bar{t}$ production density matrices that are odd under interchange of the $t$ and $\bar{t}$. In the SM these correlations, which receive no contributions from the $g g$ initial state and no contributions, at LO QCD, from $q \bar{q}$ initial states, are expected to be small. They receive NP contributions proportional to the anomalous couplings $\hat{c}_{A A}$ and $\hat{c}_{2}$.

6. As long as one considers CP-invariant interactions, $C(r, k)=C(k, r)$. A non-zero difference $C(r, k)-C(k, r)$ requires $\mathrm{P}$-even but $\mathrm{CP}$-odd absorptive contributions. In the SM at NLO there are no contributions of this type. Because our NP couplings are real and we do not take into account radiative corrections to the NP amplitudes, there are also no NP contributions to $C(r, k)-C(k, r)$ in our approximation.

7. The sums

$$
C(n, r)+C(r, n), \quad C(n, k)+C(k, n)
$$

probe P-odd, CP-even, naive T-odd absorptive (SM) contributions. The coefficients $C(n, r)$ and $C(n, k)$ receive $\mathrm{SM}$ contributions from P-odd absorptive parts of the mixed QCD-weak corrections. We have computed the respective 1-loop absorptive contributions to the $g g \rightarrow t \bar{t}$ and $q \bar{q} \rightarrow t \bar{t}$ spin density matrices. The resulting effects on (4.24) are very small, cf. tables 7 and 9. Because all anomalous couplings are real in the effective Lagrangian approach and because we do not take into account radiative corrections to the NP amplitudes, there are no NP contributions to (4.24).

8. The SM contributions referred to in the previous item cancel in

$$
C(n, r)-C(r, n), \quad C(n, k)-C(k, n) .
$$

These differences probe $\mathrm{P}$-and CP-odd (dispersive) NP contributions, that is, nonstandard CP violation. These differences in the correlation coefficients can be related to CP-odd triple correlations. Defining

$$
\mathcal{O}_{1}^{\mathrm{CP}}=\left(\hat{\ell}_{+} \times \hat{\ell}_{-}\right) \cdot \hat{\mathbf{k}}, \quad \mathcal{O}_{2}^{\mathrm{CP}}=\operatorname{sign}\left(y_{p}\right)\left(\hat{\ell}_{+} \times \hat{\ell}_{-}\right) \cdot \hat{\mathbf{r}}_{p},
$$

the following relations hold:

$$
C(n, r)-C(r, n)=9\left\langle\mathcal{O}_{1}^{\mathrm{CP}}\right\rangle, \quad C(n, k)-C(k, n)=-9\left\langle\mathcal{O}_{2}^{\mathrm{CP}}\right\rangle .
$$

Alternatively one may consider associated $\mathrm{CP}$ asymmetries, which are easier to measure. They are related to the expectation values of $(4.26)$ by $[15,95]$ :

$$
A_{i}^{\mathrm{CP}}=\frac{N_{\ell \ell}\left(\mathcal{O}_{i}^{\mathrm{CP}}>0\right)-N_{\ell \ell}\left(\mathcal{O}_{i}^{\mathrm{CP}}<0\right)}{N_{\ell \ell}}=\frac{9 \pi}{16}\left\langle\mathcal{O}_{i}^{\mathrm{CP}}\right\rangle, \quad i=1,2 .
$$

Formulae (4.27) and (4.28) hold if no cuts on the dileptonic final states other than a cut on the $t \bar{t}$ invariant mass are applied. The respective numbers in tables 7 and 9 show that $C(n, r)-C(r, n)$ is the most sensitive spin correlation observable to search 
for a top-quark chromo-electric dipole moment $\hat{d}_{t}$. The CP asymmetry $A_{1}^{\mathrm{CP}}$ is less sensitive to the CP-violating anomalous couplings than the left-hand sides of (4.27), but experimentally more robust. The measurement of the two correlations (4.27) or the two asymmetries (4.28) would allow to simultaneously determine or constrain the CEDM $\hat{d}_{t}$ and the anomalous CP-odd gluon-top-quark coupling $\hat{c}_{(--)}$.

Putting the coupling $\hat{c}_{(--)}=0$, the expectation value $\left\langle\mathcal{O}_{1}^{\mathrm{CP}}\right\rangle$ at $8 \mathrm{TeV}$ computed with the $8 \mathrm{TeV}$ value for $C(n, r)-C(r, n)$ given in table 7 agrees with the result of [15].

9. As already emphasized above, for $p p$ collisions a classification of observables with respect to $\mathrm{CP}$ is, strictly speaking, not possible because $|p p\rangle$ is not a $\mathrm{CP}$ eigenstate. However, there will be no SM contributions from $q \bar{q}$ and $g g$ initial states to the CPodd observables listed in the previous item and in (4.30) - assuming no acceptance cuts or cuts which are CP-invariant. Moreover, within our approximation we found no contribution from P-violating but CP-even terms induced by $g q$ production.

10. As to the longitudinal top-polarization coefficients $B(k), B(r)$ : the sums

$$
B_{1}(k)+B_{2}(k), \quad B_{1}(r)+B_{2}(r), \quad B_{1}\left(k^{*}\right)+B_{2}\left(k^{*}\right), \quad B_{1}\left(r^{*}\right)+B_{2}\left(r^{*}\right)
$$

receive contributions from the $\mathrm{P}$-odd weak-interaction corrections, that is, from the P-odd terms of the NLOW corrections. However, the SM-induced $t$ and $\bar{t}$ polarizations are very small to tiny, as the numbers in table 8 and 10 show. (The SM result for $B_{1}(k)+B_{2}(k)$ at $8 \mathrm{TeV}$ listed in table 8 agrees with the result computed in [15].) The sums (4.29) receive also contributions from the P-odd NP four-quark operators. Thus, the measurement of the four polarization observables (4.29) would allow to simultaneously determine or constrain four anomalous four-quark couplings, for instance $\hat{c}_{V A}, \hat{c}_{A V}, \hat{c}_{2}$, and $\hat{c}_{3}$, assuming that input on $\hat{c}_{1}$ is provided from the measurement of the four CP-even spin correlations, see item 3 above.

In the differences

$$
B_{1}(k)-B_{2}(k), \quad B_{1}(r)-B_{2}(r), \quad B_{1}\left(k^{*}\right)-B_{2}\left(k^{*}\right), \quad B_{1}\left(r^{*}\right)-B_{2}\left(r^{*}\right),
$$

which are $\mathrm{P}$ - and $\mathrm{CP}$-odd and $T_{N}$-even, the $\mathrm{SM}$ contributions to $B_{1,2}$ cancel. Nonzero differences require, in addition to $\mathrm{CP}$-violating $\mathrm{NP}$ contributions, also absorptive parts. Because our anomalous couplings are real and we do not take into account radiative corrections to the NP contributions, these differences remain zero within our approach. In [15] the chromo moments were interpreted as form factors which may have an absorptive part, and the differences of the longitudinal $t$ and $\bar{t}$ polarizations were computed in terms of $\operatorname{Im} \hat{d}_{t}$.

11. As to the combinations

$$
B_{1}(n) \pm B_{2}(n) .
$$

The sum is generated by $\mathrm{P}$ - and $\mathrm{CP}$-even absorptive terms in the differential cross section. In the SM such terms arise from a number of one-loop amplitudes for $g g, q \bar{q} \rightarrow$ 
$t \bar{t}$ which involve parton, weak gauge-boson, Higgs boson, and photon exchange. The QCD contributions given in tables 8 are in accord with the $8 \mathrm{TeV}$ result of [15]. (In this work, the vector perpendicular to the scattering plane was used in un-normalized form and a different PDF set was employed.) In addition we list in tables 8 and 10 also our results for the electroweak contributions, which arise from the absorptive parts of the mixed QCD-weak interaction and QCD-QED contributions to $g g, q \bar{q} \rightarrow t \bar{t}$. They turn out to be non-negligible in comparison to the QCD contribution. Thus, for the inclusive $t \bar{t}$ sample, the transverse polarization of the ensemble of $t$ and $\bar{t}$ quarks with respect to the reference axis $\operatorname{sign}\left(y_{p}\right) \hat{\mathbf{n}}_{p}$ is $P_{n t}=\bar{P}_{n \bar{t}} \simeq 0.5 \%$. In the difference $B_{1}(n)-B_{2}(n)$ the SM contributions cancel. The difference is sensitive to P-even, but CP-odd dispersive NP contributions, which in the framework of our effective Lagrangian approach are generated at Born level by the operator $\mathcal{O}_{(-+)}^{g}$ defined in (3.5).

12. Finally we add a remark on the scale dependence of our SM predictions for the correlation and polarization observables. As the numbers given in tables 8 and 10 show, the parity-odd combinations of the $B$ coefficients are very small in the SM but exhibit large scale variations relative to the values for $\mu=m_{t}$. The reason for this is as follows. For the numerators $N$ of the $B$ coefficients that are generated by the P-odd weak interaction contributions, one gets $N\left(\mu=2 m_{t}\right)>N\left(\mu=m_{t}\right)>N\left(\mu=m_{t} / 2\right)$, due to factorization-scale dependent logarithms in some of the $q g$ induced mixed QCD-weak corrections [100], while for the denominators $D$ of the $B$ coefficients, which are taken into account at lowest order QCD, one has $D\left(\mu=2 m_{t}\right)<D(\mu=$ $\left.m_{t}\right)<D\left(\mu=m_{t} / 2\right)$. This produces the large scale variations in the ratios $B=N / D$. The P- and CP-even correlation observables given in tables 7 and 9 are generated predominantly by QCD. Here the scale variations are moderate to small and, as is well known, the Taylor expansion (4.22) reduces the scale dependence of the results at NLO QCD with respect to LO. The weak interaction contributions with their rather large scale dependence do not spoil this because they are small compared to the QCD contributions. The correlation $C(r, r)$ is special in that there are cancellations among separate contributions, keeping $C(r, r)$ rather small and more sensitive to scale variations than $C(n, n)$ and $C(k, k)$.

The above spin-correlation and polarization observables probe all entries of the topspin dependent parts of the $t \bar{t}$ production density matrices. In addition, the various NP contributions are disentangled to a large extent, as the above discussion and tables show. Moreover, a measurement of this set of spin observables - and experimental results on $A_{\mathrm{C}}$, which yields information on the coupling $\hat{c}_{A A}$ - would provide sufficient input for determining all anomalous couplings of the effective Lagrangian (3.22). As mentioned above, the spin correlations $C\left(r^{*}, k\right), C\left(k, r^{*}\right)$, and $C\left(k, k^{*}\right)$ are also sensitive to $\hat{c}_{A A}$.

An important issue is the range of validity of the linear approximation for the NP contributions to the correlation and polarization observables. In analogy to the procedure we applied to the $t \bar{t}$ cross section and the charge asymmetry $A_{\mathrm{C}}$ in section 4.1 , - cf. eq. (4.2) and table 3 - we estimate the maximal difference between (future) experimental 


\begin{tabular}{|c|c|c|}
\hline Observable & no cut & $m_{t \bar{t}}<500 \mathrm{GeV}$ \\
\hline$\delta C(n, n)$ & 0.31 & 0.33 \\
\hline$\delta C(r, r)$ & 0.44 & 0.44 \\
\hline$\delta C(k, k)$ & 0.27 & 0.22 \\
\hline$\delta[C(r, k)+C(k, r)]$ & 0.15 & 0.19 \\
\hline$\delta[C(n, r)-C(r, n)]$ & 0.74 & 0.68 \\
\hline$\delta[C(n, k)-C(k, n)]$ & 0.34 & 0.28 \\
\hline$\delta\left[B_{1}(r)+B_{2}(r)\right]$ & $2.4 \times 10^{-2}$ & $1.5 \times 10^{-2}$ \\
\hline$\delta\left[B_{1}(k)+B_{2}(k)\right]$ & 0.18 & $7.5 \times 10^{-2}$ \\
\hline$\delta\left[B_{1}\left(r^{*}\right)+B_{2}\left(r^{*}\right)\right]$ & 0.13 & 0.11 \\
\hline$\delta\left[B_{1}\left(k^{*}\right)+B_{2}\left(k^{*}\right)\right]$ & 0.14 & $8.3 \times 10^{-2}$ \\
\hline$\delta\left[B_{1}(n)-B_{2}(n)\right]$ & 0.49 & 0.28 \\
\hline
\end{tabular}

Table 11. Moduli of maximal differences between future experimental results and SM predictions, for the correlation and polarization observables at the LHC $(13 \mathrm{TeV})$, for which the linear approximation can be applied.

measurements and SM prediction of an observable by adding the moduli of the products of the respective contributions given in tables 7-10 and the maximal values of the anomalous couplings, derived in section 3.3, for which the linear approximation can be applied. The results of these estimates are given, for the LHC $(13 \mathrm{TeV})$ in table 11 . The estimates for 8 and $14 \mathrm{TeV}$ can be obtained in completely analogous fashion. The numbers of table 11 show that the range of validity of the linear approximation is quite large.

One may compute the above spin observables also for $t \bar{t}$ events with large $t \bar{t}$ invariant mass, for instance $M_{t \bar{t}}>500 \mathrm{GeV}$. While there is no computational difficulty, one should, as discussed above, in this case abandon the linear approximation in the anomalous couplings. This leads to a proliferation of NP contributions to each of the spin-correlation and polarization coefficients, which will make a comparison with (future) data rather intransparent. One may pursue a pragmatic approach, namely, analyze only selected observables beyond the linear approximation, in particular those which turn out to be measurable with moderate or small experimental uncertainties. Such an analysis is beyond the scope of this paper and will be left to a future investigation.

\section{$5 \quad$ Summary and conclusions}

We have defined a set of correlation and polarization observables with which hadronic $t \bar{t}$ production dynamics can be probed in detail, that is, all entries of the top-spin dependent parts of the hadronic $t \bar{t}$ production density matrices, in dileptonic and semileptonic $t \bar{t}$ events at the LHC. Our observables allow, in particular, to disentangle contributions from interactions which conserve/violate parity and/or CP. We have computed these observables within the Standard Model at NLO QCD including the mixed QCD-(electro)weak 
contributions. In addition we have analyzed possible new physics contributions in terms of an effective Lagrangian that contains all gauge-invariant effective interactions of mass dimension $\leq 6$ being relevant for the parton processes at hand. Existing constraints on anomalous couplings which describe the strength of these effective NP interactions justify that only NP terms linear in the anomalous couplings are taken into account in the computation of inclusive (spin) observables for the LHC. Irrespective of these constraints we have estimated the range of validity of the linear approximation for the observables considered in this paper. Some of our polarization and spin correlation observables allow for direct searches of non-standard $\mathrm{P}$ - and $\mathrm{CP}$ violation in $t \bar{t}$ events and will provide, once measurements will have been made, direct information on respective interactions which may affect hadronic $t \bar{t}$ production. The measurement of the complete set of our inclusive spin observables, including $A_{\mathrm{C}}$, would allow to determine, respectively constrain all anomalous couplings contained in $\mathcal{L}_{\mathrm{NP}}$. Instead of $A_{\mathrm{C}}$, one may consider the observables $C\left(r^{*}, k\right)$, $C\left(k, r^{*}\right)$, and $C\left(k, k^{*}\right)$ which are the spin correlation analogues of the charge asymmetry.

Eventually, it will also be of interest to compute and measure the above $t \bar{t}$ spin correlations and $t$ and $\bar{t}$ polarizations in the kinematic regime where the energy-momentum transfer to the $t \bar{t}$ system is large, for instance for $t \bar{t}$ events with $M_{t \bar{t}}>500 \mathrm{GeV}$. In this regime the contributions of the effective NP interactions grow in general, due to their non-renormalizable nature. In this kinematic regime one should abandon the linear approximation, but then each observable receives a large number of contributions from the anomalous interactions contained in $\mathcal{L}_{\mathrm{NP}}$. Such an analysis should be done at some point for selected observables, once experimental feedback on measurement precisions will be available.

\section{A NP contributions to the $t \bar{t}$ spin density matrices}

The $t \bar{t}$ production spin density matrices to the parton reactions $g g, q \bar{q} \rightarrow t \bar{t}(g)$ and $g q(\bar{q}) \rightarrow$ $t \bar{t} q(\bar{q})$ were computed at NLO QCD (dispersive contributions) in $[12,13]$, the contributions of the NLO QCD absorptive parts were determined in [96, 97] and the contributions of the mixed QCD-weak corrections (dispersive contributions) in [98-100]. The spin-independent term of the mixed QCD-weak corrections to $q \bar{q}, g g \rightarrow t \bar{t}$, i.e., the respective contributions to the coefficient functions $A^{I}$ of eq. (2.4), were computed also in [101, 102] and, without the infrared-divergent box contributions to $q \bar{q} \rightarrow t \bar{t}$ and the corresponding real gluon radiation, in [103], and these results agree with ours. In addition, we have computed the contributions of the absorptive parts of the mixed QCD electroweak corrections. Here we do not list the latter contributions, for the sake of brevity and because they induce only very small effects (see section 4.2). Semileptonic and non-leptonic decays of polarized top-quarks are incorporated at NLO QCD [80].

Here we list the contributions to the $t \bar{t}$ spin density matrices (2.4) in the decomposition (2.6), (2.7) of the NP interactions described by the effective Lagrangian (3.22). As emphasized above, we take into account only the interferences with the tree-level QCD amplitudes of $g g, q \bar{q} \rightarrow t \bar{t}$, i.e., only terms linear in the anomalous couplings. We recall that all anomalous couplings are real dimensionless parameters. 
In the following, $s=\left(p_{1}+p_{2}\right)^{2}$, where $p_{1,2}$ are the 4-momenta of the initial partons. Furthermore we define (cf. (2.5))

$$
z=\frac{2 m_{t}}{\sqrt{s}}, \quad \beta=\sqrt{1-z^{2}}, \quad y=\hat{\mathbf{p}} \cdot \hat{\mathbf{k}} .
$$

\section{A.1 NP contributions to $g g \rightarrow t \bar{t}$}

For the non-vanishing NP contributions to the coefficients of the $g g \rightarrow t \bar{t}$ spin density matrix defined in $(2.6),(2.7)$ we get in the linear approximation, dropping the superscript $I=g g$ :

$$
\begin{aligned}
& A=\frac{N_{c}^{2}\left(1+\beta^{2} y^{2}\right)-2}{1-\beta^{2} y^{2}} \hat{\mu}_{t}, \\
& b_{n}^{+}=-b_{n}^{-}=2 N_{c}^{2} \frac{y \beta^{2}}{z} \frac{\sqrt{1-y^{2}}}{\left(1-\beta^{2} y^{2}\right)} \hat{c}_{(-+)}, \\
& c_{r r}=\frac{N_{c}^{2}\left[-1+\beta^{4} y^{2}\left(1-y^{2}\right)^{2}+\beta^{2}\left(1-y^{2}+y^{4}\right)\right]+2-2 \beta^{2}\left(1-y^{2}+y^{4}\right)}{\left(1-\beta^{2} y^{2}\right)^{2}} \hat{\mu}_{t}, \\
& c_{k k}=N_{c}^{2} \frac{-1-\beta^{2}\left(-2+y^{4}\right)+\beta^{6} y^{2}\left(-1-y^{2}+y^{4}\right)+\beta^{4}\left(-1+y^{2}+2 y^{4}-y^{6}\right)}{z^{2}\left(1-\beta^{2} y^{2}\right)^{2}} \hat{\mu}_{t} \\
& +\frac{2}{z^{2}} \frac{1+\beta^{4}\left(1+y^{2}-y^{4}\right)+\beta^{2}\left(-2-y^{2}+y^{4}\right)}{\left(1-\beta^{2} y^{2}\right)^{2}} \hat{\mu}_{t}, \\
& c_{n n}=\frac{2-N_{c}^{2}}{1-\beta^{2} y^{2}} \hat{\mu}_{t} \\
& c_{r k}=N_{c}^{2} \frac{y \sqrt{1-y^{2}}}{2 z} \frac{-2 \beta^{2} y^{2}+\beta^{6} y^{2}\left(-1+y^{2}\right)+\beta^{4}\left(1+3 y^{2}-2 y^{4}\right)}{\left(1-\beta^{2} y^{2}\right)^{2}} \hat{\mu}_{t} \\
& -\frac{y \beta^{2} \sqrt{1-y^{2}}}{z} \frac{1+y^{2}\left(-2+\beta^{2}\right)}{\left(1-\beta^{2} y^{2}\right)^{2}} \hat{\mu}_{t}, \\
& c_{r}=-\frac{N_{c}^{2} y \beta}{z^{3}} \frac{\sqrt{1-y^{2}}\left(1-3 z^{4}+\beta^{2}\left(z^{2}-z^{4} y^{2}\right)+\beta^{6}\left(y^{2}+y^{4}\right)-\beta^{4}\left(1+y^{2}+y^{2} z^{2}+y^{4}\right)\right)}{\left(1-\beta^{2} y^{2}\right)^{2}} \hat{c}_{(--)} \\
& -\frac{N_{c}^{2} y \beta \sqrt{1-y^{2}}}{2 z} \frac{2+\beta^{2}\left(-3+y^{2}\right)+\beta^{4} y^{2}\left(-1+y^{2}\right)}{\left(1-y^{2} \beta^{2}\right)^{2}} \hat{d}_{t} \\
& -2 \frac{y \beta}{z} \frac{\sqrt{1-y^{2}}\left(1+\beta^{2}\left(y^{2}-2\right)\right)}{\left(1-\beta^{2} y^{2}\right)^{2}} \hat{c}_{(--)}+\frac{y \beta \sqrt{1-y^{2}}}{z} \frac{1+\beta^{2}\left(-2+y^{2}\right)}{\left(1-\beta^{2} y^{2}\right)^{2}} \hat{d}_{t}, \\
& c_{k}=-\frac{N_{c}^{2} \beta}{z^{2}\left(1-\beta^{2} y^{2}\right)^{2}}\left[-1+y^{2}+z^{4}\left(2-3 y^{2}\right)+\beta^{6} y^{4}\left(-1+y^{2}\right)-\beta^{2}\left(-1+y^{2}-2 y^{2} z^{4}+y^{4} z^{4}\right)\right. \\
& \left.+\beta^{4}\left(y^{4}-y^{6}\right)\right] \hat{c}_{(--)}-\frac{N_{c}^{2} \beta}{2} \frac{-3+2 y^{2}+\beta^{2}\left(2-3 y^{2}+y^{4}\right)+\beta^{4} y^{2}\left(2-2 y^{2}+y^{4}\right)}{\left(1-\beta^{2} y^{2}\right)^{2}} \hat{d}_{t} \\
& +2 \beta\left(1-y^{2}\right) \frac{1+\beta^{2}\left(-2+y^{2}\right)}{\left(1-\beta^{2} y^{2}\right)^{2}} \hat{c}_{(--)}+\beta \frac{-3+y^{2}+\beta^{2}\left(2-y^{2}+y^{4}\right)}{\left(1-\beta^{2} y^{2}\right)^{2}} \hat{d}_{t} .
\end{aligned}
$$




\section{A.2 NP contributions to $q \bar{q} \rightarrow t \bar{t}$}

For the non-vanishing NP contributions to the coefficients of the $q \bar{q} \rightarrow t \bar{t}$ spin density matrix defined in (2.6), (2.7) we get in the linear approximation, dropping the superscript $I=q \bar{q}$ :

$$
\begin{aligned}
A & =\frac{2+\beta^{2}\left(-1+y^{2}\right)}{4 z^{2}}\left[\hat{c}_{V V} \pm \frac{\left.\hat{c}_{1}\right]}{2}\right]+\frac{y \beta}{2 z^{2}}\left[\hat{c}_{A A} \pm \frac{\hat{c}_{2}}{2}\right]+\frac{1}{2} \hat{\mu}_{t} \\
b_{r}^{+} & =b_{r}^{-}=\frac{y \beta \sqrt{1-y^{2}}}{4 z}\left[\hat{c}_{V A} \pm \frac{\hat{c}_{3}}{2}\right]+\frac{\sqrt{1-y^{2}}}{2 z}\left[\hat{c}_{A V} \pm \frac{\hat{c}_{1}}{2} \mp \frac{\hat{c}_{2}}{2} \pm \frac{\hat{c}_{3}}{2}\right], \\
b_{k}^{+} & =b_{k}^{-}=\frac{\beta\left(1+y^{2}\right)}{4 z^{2}}\left[\hat{c}_{V A} \pm \frac{\hat{c}_{3}}{2}\right]+\frac{y}{2 z^{2}}\left[\hat{c}_{A V} \pm \frac{\hat{c}_{1}}{2} \mp \frac{\hat{c}_{2}}{2} \pm \frac{\hat{c}_{3}}{2}\right] \\
c_{r r} & =-\frac{\left(2-\beta^{2}\right)\left(1-y^{2}\right)}{4 z^{2}}\left[\hat{c}_{V V} \pm \frac{\hat{c}_{1}}{2}\right]+\frac{1}{2}\left(1-y^{2}\right) \hat{\mu}_{t} \\
c_{k k} & =\frac{\left(2 y^{2}+\beta^{2}\left(1-y^{2}\right)\right)}{4 z^{2}}\left[\hat{c}_{V V} \pm \frac{\hat{c}_{1}}{2}\right]+\frac{y \beta}{2 z^{2}}\left[\hat{c}_{A A} \pm \frac{\hat{c}_{2}}{2}\right]+\frac{y^{2}}{2} \hat{\mu}_{t} \\
c_{n n} & =\frac{\beta^{2}\left(-1+y^{2}\right)}{4 z^{2}}\left[\hat{c}_{V V} \pm \frac{\hat{c}_{1}}{2}\right], \\
c_{r k} & =\frac{y \sqrt{1-y^{2}}}{2 z}\left[\hat{c}_{V V} \pm \frac{\hat{c}_{1}}{2}\right]+\frac{\beta \sqrt{1-y^{2}}}{4 z}\left[\hat{c}_{A A} \pm \frac{\hat{c}_{2}}{2}\right]+\frac{y \sqrt{1-y^{2}}\left(2-\beta^{2}\right)}{4 z} \hat{\mu}_{t}, \\
c_{r} & =-\frac{y \beta \sqrt{1-y^{2}}}{2 z} \hat{c}_{(--)}+\frac{y \beta \sqrt{1-y^{2}}}{4 z} \hat{d}_{t} \\
c_{k} & =\frac{1}{2} \beta\left(1-y^{2}\right) \hat{c}_{(--)}+\frac{\beta}{4}\left(-1+z^{2}\right) \hat{d}_{t} .
\end{aligned}
$$

The terms proportional to $\hat{\mu}_{t}, \hat{d}_{t}$, and $\hat{c}_{(--)}$apply to all $q \neq t$. The terms proportional to the couplings $\hat{c}_{X}$ apply only to the first quark generation; the upper (lower) sign refers to $u \bar{u}(d \bar{d})$ in the initial state.

\section{Acknowledgments}

We wish to thank C. Deterre, J. Howarth, M. Levy, G. McGoldrick, Y. Peters, M. Rudolph, and R. Schäfer of the ATLAS Top-quark spin analysis group, and C. Schwanenberger for continuing discussions. The work of W.B. was supported by BMBF and that of Z.G. Si by NSFC and by Natural Science Foundation of Shandong Province. D.H. is supported by Deutsche Forschungsgemeinschaft through Graduiertenkolleg GRK 1675.

Open Access. This article is distributed under the terms of the Creative Commons Attribution License (CC-BY 4.0), which permits any use, distribution and reproduction in any medium, provided the original author(s) and source are credited. 


\section{References}

[1] CDF collaboration, T. Aaltonen et al., Measurement of $t \bar{t}$ Spin Correlation in p $\bar{p}$ Collisions Using the CDF II Detector at the Tevatron, Phys. Rev. D 83 (2011) 031104 [arXiv: 1012.3093] [INSPIRE].

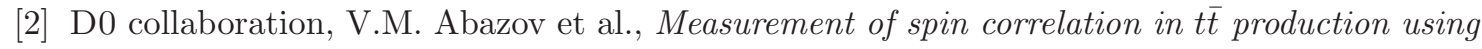
a matrix element approach, Phys. Rev. Lett. 107 (2011) 032001 [arXiv:1104.5194] [INSPIRE].

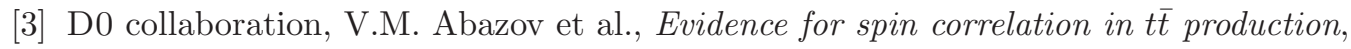
Phys. Rev. Lett. 108 (2012) 032004 [arXiv:1110.4194] [INSPIRE].

[4] D0 collaboration, V.M. Abazov et al., Measurement of Leptonic Asymmetries and Top Quark Polarization in $t \bar{t}$ Production, Phys. Rev. D 87 (2013) 011103 [arXiv: 1207.0364] [INSPIRE].

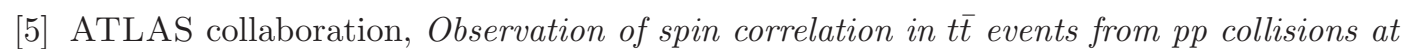
$\sqrt{s}=7$ TeV using the ATLAS detector, Phys. Rev. Lett. 108 (2012) 212001 [arXiv:1203.4081] [INSPIRE].

[6] ATLAS collaboration, Measurement of Top Quark Polarization in Top-Antitop Events from Proton-Proton Collisions at $\sqrt{s}=7$ TeV Using the ATLAS Detector,

Phys. Rev. Lett. 111 (2013) 232002 [arXiv:1307.6511] [INSPIRE].

[7] CMS collaboration, Measurements of $t \bar{t}$ spin correlations and top-quark polarization using dilepton final states in pp collisions at $\sqrt{s}=7$ TeV, Phys. Rev. Lett. 112 (2014) 182001 [arXiv: 1311.3924] [INSPIRE].

[8] ATLAS collaboration, Measurements of spin correlation in top-antitop quark events from proton-proton collisions at $\sqrt{s}=7$ TeV using the ATLAS detector, Phys. Rev. D 90 (2014) 112016 [arXiv:1407.4314] [InSPIRE].

[9] CMS Collaboration, Search for Anomalous Top Chromomagnetic Dipole Moments from angular distributions in $t \bar{t}$ Dileptonic events at $\sqrt{s}=7$ TeV with the CMS detector, CMS-PAS-TOP-14-005 (2014) [INSPIRE].

[10] ATLAS collaboration, Measurement of Spin Correlation in Top-Antitop Quark Events and Search for Top Squark Pair Production in pp Collisions at $\sqrt{s}=8$ TeV Using the ATLAS Detector, Phys. Rev. Lett. 114 (2015) 142001 [arXiv: 1412.4742] [INSPIRE].

[11] CMS Collaboration, Measurement of spin correlations in top pair events in the lepton + jets channel with the matrix element method at 8 TeV, CMS-PAS-TOP-13-015 (2015) [INSPIRE].

[12] W. Bernreuther, A. Brandenburg, Z.-G. Si and P. Uwer, Top quark spin correlations at hadron colliders: Predictions at next-to-leading order $Q C D$,

Phys. Rev. Lett. 87 (2001) 242002 [hep-ph/0107086] [INSPIRE].

[13] W. Bernreuther, A. Brandenburg, Z.-G. Si and P. Uwer, Top quark pair production and decay at hadron colliders, Nucl. Phys. B 690 (2004) 81 [hep-ph/0403035] [INSPIRE].

[14] W. Bernreuther and Z.-G. Si, Distributions and correlations for top quark pair production and decay at the Tevatron and LHC, Nucl. Phys. B 837 (2010) 90 [arXiv:1003.3926] [INSPIRE].

[15] W. Bernreuther and Z.-G. Si, Top quark spin correlations and polarization at the LHC: standard model predictions and effects of anomalous top chromo moments, Phys. Lett. B 725 (2013) 115 [Corrigendum ibid. 744 (2015) 413] [arXiv:1305.2066] [INSPIRE]. 
[16] W. Buchmüller and D. Wyler, Effective Lagrangian Analysis of New Interactions and Flavor Conservation, Nucl. Phys. B 268 (1986) 621 [InSPIRE].

[17] B. Grzadkowski, M. Iskrzynski, M. Misiak and J. Rosiek, Dimension-Six Terms in the Standard Model Lagrangian, JHEP 10 (2010) 085 [arXiv: 1008.4884] [INSPIRE].

[18] J.A. Aguilar-Saavedra, A Minimal set of top anomalous couplings, Nucl. Phys. B 812 (2009) 181 [arXiv:0811.3842] [InSPIRE].

[19] J.A. Aguilar-Saavedra, A Minimal set of top-Higgs anomalous couplings, Nucl. Phys. B 821 (2009) 215 [arXiv:0904.2387] [INSPIRE].

[20] C. Zhang and S. Willenbrock, Effective-Field-Theory Approach to Top-Quark Production and Decay, Phys. Rev. D 83 (2011) 034006 [arXiv: 1008.3869] [InSPIRE].

[21] J.A. Aguilar-Saavedra, Effective four-fermion operators in top physics: A Roadmap, Nucl. Phys. B 843 (2011) 638 [Erratum ibid. B 851 (2011) 443] [arXiv:1008.3562] [INSPIRE].

[22] C. Degrande, J.-M. Gerard, C. Grojean, F. Maltoni and G. Servant, Non-resonant New Physics in Top Pair Production at Hadron Colliders, JHEP 03 (2011) 125 [arXiv: 1010.6304] [INSPIRE].

[23] D. Atwood, A. Aeppli and A. Soni, Extracting anomalous gluon-top effective couplings at the supercolliders, Phys. Rev. Lett. 69 (1992) 2754 [INSPIRE].

[24] A. Brandenburg and J.P. Ma, CP odd observables for the top-antitop system produced at proton-antiproton and proton-proton colliders, Phys. Lett. B 298 (1993) 211 [INSPIRE].

[25] D. Atwood, A. Kagan and T.G. Rizzo, Constraining anomalous top quark couplings at the Tevatron, Phys. Rev. D 52 (1995) 6264 [hep-ph/9407408] [INSPIRE].

[26] P. Haberl, O. Nachtmann and A. Wilch, Top production in hadron hadron collisions and anomalous top-gluon couplings, Phys. Rev. D 53 (1996) 4875 [hep-ph/9505409] [inSPIRE].

[27] K.-m. Cheung, Probing the chromoelectric and chromomagnetic dipole moments of the top quark at hadronic colliders, Phys. Rev. D 53 (1996) 3604 [hep-ph/9511260] [INSPIRE].

[28] K.-m. Cheung, Probing nonstandard top couplings using spin correlation, Phys. Rev. D 55 (1997) 4430 [hep-ph/9610368] [INSPIRE].

[29] B. Grzadkowski, B. Lampe and K.J. Abraham, CP violation, top quarks and the Tevatron upgrade, Phys. Lett. B 415 (1997) 193 [hep-ph/9706489] [INSPIRE].

[30] J.M. Yang and B.-L. Young, Dimension-six CP-violating operators of the third family quarks and their effects at colliders, Phys. Rev. D 56 (1997) 5907 [hep-ph/9703463] [INSPIRE].

[31] K.-i. Hikasa, K. Whisnant, J.M. Yang and B.-L. Young, Probing anomalous top quark interactions at the Fermilab Tevatron collider, Phys. Rev. D 58 (1998) 114003 [hep-ph/9806401] [INSPIRE].

[32] H.-Y. Zhou, CP violation in top quark pair production at hadron colliders, Phys. Rev. D 58 (1998) 114002 [hep-ph/9805358] [InSPIRE].

[33] D. Atwood, S. Bar-Shalom, G. Eilam and A. Soni, CP violation in top physics, Phys. Rept. 347 (2001) 1 [hep-ph/0006032] [INSPIRE].

[34] R. Martinez and J.A. Rodriguez, The Anomalous chromomagnetic dipole moment of the top quark in the standard model and beyond, Phys. Rev. D 65 (2002) 057301 [hep-ph/0109109] [INSPIRE]. 
[35] B. Lillie, J. Shu and T.M.P. Tait, Top Compositeness at the Tevatron and LHC, JHEP 04 (2008) 087 [arXiv: 0712.3057] [INSPIRE].

[36] S.K. Gupta and G. Valencia, CP-odd correlations using jet momenta from t $\bar{t}$ events at the Tevatron, Phys. Rev. D 81 (2010) 034013 [arXiv:0912.0707] [InSPIRE].

[37] S.K. Gupta, A.S. Mete and G. Valencia, CP violating anomalous top-quark couplings at the LHC, Phys. Rev. D 80 (2009) 034013 [arXiv: 0905.1074] [INSPIRE].

[38] Z. Hioki and K. Ohkuma, Search for anomalous top-gluon couplings at LHC revisited, Eur. Phys. J. C 65 (2010) 127 [arXiv: 0910.3049] [inSPIRE].

[39] Z. Hioki and K. Ohkuma, Exploring anomalous top interactions via the final lepton in $t \bar{t}$ productions/decays at hadron colliders, Phys. Rev. D 83 (2011) 114045 [arXiv:1104.1221] [INSPIRE].

[40] D. Choudhury and P. Saha, Probing Top Anomalous Couplings at the Tevatron and the Large Hadron Collider, Pramana 77 (2011) 1079 [arXiv:0911.5016] [INSPIRE].

[41] J. Cao, K. Hikasa, L. Wang, L. Wu and J.M. Yang, Testing new physics models by top charge asymmetry and polarization at the LHC, Phys. Rev. D 85 (2012) 014025 [arXiv:1109.6543] [INSPIRE].

[42] J.F. Kamenik, M. Papucci and A. Weiler, Constraining the dipole moments of the top quark, Phys. Rev. D 85 (2012) 071501 [arXiv:1107.3143] [InSPIRE].

[43] F. Bach and T. Ohl, Anomalous Top Couplings at Hadron Colliders Revisited, Phys. Rev. D 86 (2012) 114026 [arXiv:1209.4564] [INSPIRE].

[44] E. Gabrielli, A. Racioppi, M. Raidal and H. Veermae, Implications of effective axial-vector coupling of gluon for $t \bar{t}$ spin polarizations at the LHC, Phys. Rev. D 87 (2013) 054001 [arXiv: 1212.3272] [INSPIRE].

[45] Z. Hioki and K. Ohkuma, Optimal-observable Analysis of Possible Non-standard Top-quark Couplings in $p p \rightarrow t \bar{t} X \rightarrow l^{+} X^{\prime}$, Phys. Lett. B 716 (2012) 310 [arXiv:1206.2413] [INSPIRE].

[46] C. Englert, A. Freitas, M. Spira and P.M. Zerwas, Constraining the Intrinsic Structure of Top-Quarks, Phys. Lett. B 721 (2013) 261 [arXiv:1210.2570] [INSPIRE].

[47] S.S. Biswal, S.D. Rindani and P. Sharma, Probing chromomagnetic and chromoelectric couplings of the top quark using its polarization in pair production at hadron colliders, Phys. Rev. D 88 (2013) 074018 [arXiv:1211.4075] [InSPIRE].

[48] M. Baumgart and B. Tweedie, A New Twist on Top Quark Spin Correlations, JHEP 03 (2013) 117 [arXiv: 1212.4888] [INSPIRE].

[49] S.Y. Ayazi, H. Hesari and M.M. Najafabadi, Probing the top quark chromoelectric and chromomagnetic dipole moments in single top $t W$-channel at the $L H C$, Phys. Lett. B 727 (2013) 199 [arXiv:1307.1846] [INSPIRE].

[50] Z. Hioki and K. Ohkuma, Latest constraint on nonstandard top-gluon couplings at hadron colliders and its future prospect, Phys. Rev. D 88 (2013) 017503 [arXiv:1306.5387] [INSPIRE].

[51] M. Fabbrichesi, M. Pinamonti and A. Tonero, Stringent limits on top-quark compositeness from $t \bar{t}$ production at the Tevatron and the LHC, Phys. Rev. D 89 (2014) 074028 [arXiv: 1307.5750] [INSPIRE].

[52] G. Bélanger, R.M. Godbole, L. Hartgring and I. Niessen, Top Polarization in Stop Production at the LHC, JHEP 05 (2013) 167 [arXiv: 1212.3526] [INSPIRE]. 
[53] A. Hayreter and G. Valencia, Constraints on anomalous color dipole operators from Higgs boson production at the LHC, Phys. Rev. D 88 (2013) 034033 [arXiv: 1304.6976] [INSPIRE].

[54] M. König, M. Neubert and D.M. Straub, Dipole operator constraints on composite Higgs models, Eur. Phys. J. C 74 (2014) 2945 [arXiv: 1403.2756] [INSPIRE].

[55] J.A. Aguilar-Saavedra, B. Fuks and M.L. Mangano, Pinning down top dipole moments with ultra-boosted tops, Phys. Rev. D 91 (2015) 094021 [arXiv:1412.6654] [INSPIRE].

[56] I.D. Choudhury and A. Lahiri, Anomalous chromomagnetic moment of quarks, Mod. Phys. Lett. A 30 (2015) 1550113 [arXiv:1409.0073] [InSPIRE].

[57] K. Kiers, P. Saha, A. Szynkman, D. London, S. Judge and J. Melendez, Search for New Physics in Rare Top Decays: $t \bar{t}$ Spin Correlations and Other Observables, Phys. Rev. D 90 (2014) 094015 [arXiv:1407.1724] [INSPIRE].

[58] A.V. Prasath, R.M. Godbole and S.D. Rindani, Longitudinal top polarisation measurement and anomalous Wtb coupling, Eur. Phys. J. C 75 (2015) 402 [arXiv:1405.1264] [INSPIRE].

[59] B. Tweedie, Better Hadronic Top Quark Polarimetry, Phys. Rev. D 90 (2014) 094010 [arXiv: 1401.3021] [INSPIRE].

[60] A. Carmona et al., From Tevatron's top and lepton-based asymmetries to the LHC, JHEP 07 (2014) 005 [arXiv: 1401.2443] [INSPIRE].

[61] C. Zhang, Effective field theory approach to top-quark decay at next-to-leading order in QCD, Phys. Rev. D 90 (2014) 014008 [arXiv: 1404.1264] [INSPIRE].

[62] H. Hesari and M. Mohammadi Najafabadi, Direct photon production as a probe of quarks chromoelectric and chromomagnetic dipole moments at the LHC, Phys. Rev. D 91 (2015) 057502 [arXiv: 1407.5887] [inSPIRE].

[63] A. Kobakhidze, L. Wu and J. Yue, Anomalous Top-Higgs Couplings and Top Polarisation in Single Top and Higgs Associated Production at the LHC, JHEP 10 (2014) 100 [arXiv: 1406.1961] [INSPIRE].

[64] Z. Hioki and K. Ohkuma, Final charged-lepton angular distribution and possible anomalous top-quark couplings in $p p \rightarrow t \bar{t} X \rightarrow \ell^{+} X^{\prime}$, Phys. Lett. B 736 (2014) 1 [arXiv:1406.2475] [INSPIRE].

[65] S. Jung, P. Ko, Y.W. Yoon and C. Yu, Renormalization group-induced phenomena of top pairs from four-quark effective operators, JHEP 08 (2014) 120 [arXiv:1406.4570] [INSPIRE].

[66] R. Gaitan, E.A. Garces, J.H.M. de Oca and R. Martinez, Top quark Chromoelectric and Chromomagnetic Dipole Moments in a Two Higgs Doublet Model with CP-violation, arXiv: 1505.04168 [INSPIRE].

[67] R.M. Godbole, G. Mendiratta and S. Rindani, Looking for BSM physics using top-quark polarization and decay-lepton kinematic asymmetries, Phys. Rev. D 92 (2015) 094013 [arXiv: 1506. 07486] [INSPIRE].

[68] D. Buarque Franzosi and C. Zhang, Probing the top-quark chromomagnetic dipole moment at next-to-leading order in QCD, Phys. Rev. D 91 (2015) 114010 [arXiv:1503.08841] [INSPIRE].

[69] S.D. Rindani, P. Sharma and A.W. Thomas, Polarization of top quark as a probe of its chromomagnetic and chromoelectric couplings in $t W$ production at the Large Hadron Collider, JHEP 10 (2015) 180 [arXiv:1507.08385] [INSPIRE]. 
[70] A. Buckley et al., A global fit of top quark effective theory to data, arXiv:1506.08845 [INSPIRE].

[71] J. de Blas, M. Chala and J. Santiago, Renormalization Group Constraints on New Top Interactions from Electroweak Precision Data, JHEP 09 (2015) 189 [arXiv:1507.00757] [INSPIRE].

[72] M. Czakon, P. Fiedler and A. Mitov, Total Top-Quark Pair-Production Cross Section at Hadron Colliders Through $O\left(\alpha_{S}^{4}\right)$, Phys. Rev. Lett. 110 (2013) 252004 [arXiv:1303.6254] [INSPIRE].

[73] W. Bernreuther and A. Brandenburg, Tracing CP-violation in the production of top quark pairs by multiple TeV proton proton collisions, Phys. Rev. D 49 (1994) 4481 [hep-ph/9312210] [INSPIRE].

[74] S.D. Rindani, Effect of anomalous $t b W$ vertex on decay lepton distributions in $e^{+} e^{-} \rightarrow t \bar{t}$ and CP-violating asymmetries, Pramana 54 (2000) 791 [hep-ph/0002006] [INSPIRE].

[75] B. Grzadkowski and Z. Hioki, New hints for testing anomalous top quark interactions at future linear colliders, Phys. Lett. B 476 (2000) 87 [hep-ph/9911505] [INSPIRE].

[76] B. Grzadkowski and Z. Hioki, Decoupling of anomalous top decay vertices in angular distribution of secondary particles, Phys. Lett. B 557 (2003) 55 [hep-ph/0208079] [INSPIRE].

[77] R.M. Godbole, S.D. Rindani and R.K. Singh, Lepton distribution as a probe of new physics in production and decay of the $t$ quark and its polarization, JHEP 12 (2006) 021 [hep-ph/0605100] [INSPIRE].

[78] M. Fabbrichesi, M. Pinamonti and A. Tonero, Limits on anomalous top quark gauge couplings from Tevatron and LHC data, Eur. Phys. J. C 74 (2014) 3193 [arXiv:1406.5393] [INSPIRE].

[79] Q.-H. Cao, B. Yan, J.-H. Yu and C. Zhang, A General Analysis of Wtb anomalous Couplings, arXiv:1504.03785 [INSPIRE].

[80] A. Brandenburg, Z.-G. Si and P. Uwer, QCD corrected spin analyzing power of jets in decays of polarized top quarks, Phys. Lett. B 539 (2002) 235 [hep-ph/0205023] [INSPIRE].

[81] R. Martinez and J.A. Rodriguez, Using the radiative decay $b \rightarrow s \gamma$ to bound the chromomagnetic dipole moment of the top quark, Phys. Rev. D 55 (1997) 3212 [hep-ph/9612438] [INSPIRE].

[82] W. Hollik and M. Kollar, NLO QED contributions to top-pair production at hadron collider, Phys. Rev. D 77 (2008) 014008 [arXiv:0708.1697] [INSPIRE].

[83] H.-L. Lai et al., New parton distributions for collider physics, Phys. Rev. D 82 (2010) 074024 [arXiv:1007.2241] [InSPIRE].

[84] ATLAS collaboration, Combination of ATLAS and CMS top quark pair cross section measurements in the e $\mu$ final state using proton-proton collisions at $\sqrt{s}=8 \mathrm{TeV}$, ATLAS-CONF-2014-054 (2014) [INSPIRE].

[85] M. Czakon and A. Mitov, Top++: A Program for the Calculation of the Top-Pair Cross-Section at Hadron Colliders, Comput. Phys. Commun. 185 (2014) 2930 [arXiv:1112.5675] [INSPIRE].

[86] W. Bernreuther and Z.-G. Si, Top quark and leptonic charge asymmetries for the Tevatron and LHC, Phys. Rev. D 86 (2012) 034026 [arXiv:1205.6580] [INSPIRE]. 
[87] J.H. Kuhn and G. Rodrigo, Charge asymmetries of top quarks at hadron colliders revisited, JHEP 01 (2012) 063 [arXiv:1109.6830] [INSPIRE].

[88] CMS collaboration, Inclusive and differential measurements of the t $\bar{t}$ charge asymmetry in pp collisions at $\sqrt{s}=8 \mathrm{TeV}$, arXiv:1507.03119 [INSPIRE].

[89] M. Czakon, P. Fiedler and A. Mitov, Resolving the Tevatron Top Quark Forward-Backward Asymmetry Puzzle: Fully Differential Next-to-Next-to-Leading-Order Calculation, Phys. Rev. Lett. 115 (2015) 052001 [arXiv: 1411.3007] [INSPIRE].

[90] E. Gabrielli and M. Raidal, Effective axial-vector coupling of gluon as an explanation to the top quark asymmetry, Phys. Rev. D 84 (2011) 054017 [arXiv: 1106.4553] [INSPIRE].

[91] J.A. Aguilar-Saavedra and M. Pérez-Victoria, Probing the Tevatron t $\bar{t}$ asymmetry at LHC, JHEP 05 (2011) 034 [arXiv: 1103.2765] [INSPIRE].

[92] C. Delaunay, O. Gedalia, Y. Hochberg, G. Perez and Y. Soreq, Implications of the CDF t $\bar{t}$ Forward-Backward Asymmetry for Hard Top Physics, JHEP 08 (2011) 031 [arXiv: 1103.2297] [INSPIRE].

[93] E. Gabrielli, M. Raidal and A. Racioppi, Implications of the effective axial-vector coupling of gluon on top-quark charge asymmetry at the LHC, Phys. Rev. D 85 (2012) 074021 [arXiv:1112.5885] [INSPIRE].

[94] J.A. Aguilar-Saavedra, D. Amidei, A. Juste and M. Pérez-Victoria, Asymmetries in top quark pair production at hadron colliders, Rev. Mod. Phys. 87 (2015) 421 [arXiv:1406.1798] [INSPIRE].

[95] W. Bernreuther, A. Brandenburg and M. Flesch, Effects of Higgs sector CP-violation in top quark pair production at the LHC, hep-ph/9812387 [INSPIRE].

[96] W. Bernreuther, A. Brandenburg and P. Uwer, Transverse polarization of top quark pairs at the Tevatron and the large hadron collider, Phys. Lett. B 368 (1996) 153 [hep-ph/9510300] [INSPIRE].

[97] W.G.D. Dharmaratna and G.R. Goldstein, Single quark polarization in quantum chromodynamics subprocesses, Phys. Rev. D 53 (1996) 1073 [INSPIRE].

[98] W. Bernreuther, M. Fuecker and Z.-G. Si, Mixed QCD and weak corrections to top quark pair production at hadron colliders, Phys. Lett. B 633 (2006) 54 [hep-ph/0508091] [INSPIRE].

[99] W. Bernreuther, M. Fuecker and Z.-G. Si, Weak interaction corrections to hadronic top quark pair production, Phys. Rev. D 74 (2006) 113005 [hep-ph/0610334] [INSPIRE].

[100] W. Bernreuther, M. Fucker and Z.-G. Si, Weak interaction corrections to hadronic top quark pair production: Contributions from quark-gluon and $b \bar{b}$ induced reactions, Phys. Rev. D 78 (2008) 017503 [arXiv:0804.1237] [INSPIRE].

[101] J.H. Kuhn, A. Scharf and P. Uwer, Electroweak corrections to top-quark pair production in quark-antiquark annihilation, Eur. Phys. J. C 45 (2006) 139 [hep-ph/0508092] [INSPIRE].

[102] J.H. Kuhn, A. Scharf and P. Uwer, Electroweak effects in top-quark pair production at hadron colliders, Eur. Phys. J. C 51 (2007) 37 [hep-ph/0610335] [INSPIRE].

[103] W. Beenakker, A. Denner, W. Hollik, R. Mertig, T. Sack and D. Wackeroth, Electroweak one loop contributions to top pair production in hadron colliders,

Nucl. Phys. B 411 (1994) 343 [inSPIRE]. 